\title{
Optimal feedback strategies for bacterial growth with degradation, recycling, and effect of temperature
}

\author{
Yegorov Ivan $^{1,{ }^{*}, \text { Mairet } \text { Francis }^{2} \text {, Gouze Jean-Luc }}{ }^{1}$ \\ ${ }^{1}$ UPMC Univ Paris 06, Univ Cote dAzur, CNRS, Inria,INRA,BIOCORE Team, F-06902 Sophia Antipolis, \\ France. \\ 2 IFREMER PBA, Nantes, France. \\ *Corresponding author : Ivan Yegorov, email address : ivanyegorov@gmail.com
}

\begin{abstract}
:
Mechanisms of bacterial adaptation to environmental changes are of great interest for both fundamental biology and engineering applications. In this work, we consider a continuous-time dynamic problem of resource allocation between metabolic and gene expression machineries for a self-replicating prokaryotic cell population. In compliance with evolutionary principles, the criterion is to maximize the accumulated structural biomass. In the model, we include both the degradation of proteins into amino acids and the recycling of the latter (ie, using as precursors again). On the basis of the analytical investigation of our problem by Pontryagin's maximum principle, we develop a numerical method to approximate the switching curve of the optimal feedback control strategy. The obtained field of extremal state trajectories consists of chattering arcs and 1 steady-state singular arc. The constructed feedback control law can serve as a benchmark for comparing actual bacterial strategies of resource allocation. We also study the influence of temperature, whose increase intensifies protein degradation. While the growth rate suddenly decreases with the increase in temperature in a certain range, the optimal control synthesis appears to be essentially less sensitive.
\end{abstract}

Keywords : bacterial growth, chattering regime, effect of temperature, feedback strategy, optimal control, Pontryagin's maximum principle, protein degradation, recycling, resource allocation, singular regime, switching curve 


\section{INTRODUCTION}

For both fundamental biology and engineering applications, it is relevant to investigate how microorganisms adapt to changing environmental conditions [1-3]. In compliance with evolutionary principles, internal regulation mechanisms of bacteria are expected to maximize appropriate fitness criteria. Indeed, the results of various experimental studies appear to be in good agreement with the predictions of suitable optimization-based mathematical models [4-6]. In particular, the widely adopted principle of growth maximization (i. e., maximum accumulation of structural biomass) is followed with rather high accuracy by the ppGpp-based strategy of regulating protein synthesis in the enterobacterium Escherichia coli [7,8] (ppGpp is a signalling molecule whose concentration

Copyright (C) 2017 John Wiley \& Sons, Ltd.

Prepared using ocaauth.cls [Version: 2010/03/27 v2.00] 
affects synthesis of ribosomal proteins). In some situations, other optimization objectives can also be biologically relevant [9].

The related models are often represented as problems of optimal allocation of available resources (such as external nutrients transformed into building blocks for protein synthesis) between different cellular machineries. This can also be viewed as optimal arrangement of cellular reaction fluxes. Several studies developed large-scale network modeling frameworks [10-12], which are essentially numerical. Alternatively, it is possible to propose less computationally expensive models that focus on smaller amounts of substantial characteristics and allow wider analytical investigation, but still lead to reasonable and useful conclusions $[8,13-16]$. This approach is employed in the current work. Also note that, in comparison with purely steady-state considerations, dynamic modeling perspectives can give more realistic descriptions of bacterial growth and resource allocation $[8,12,14,15]$.

The work [8] studied a continuous-time dynamic problem of resource allocation between metabolic and gene expression machineries for a self-replicating prokaryotic cell population with the growth maximization criterion. In [17], this model was extended by taking macromolecular degradation into account. For the sake of simplicity, it was assumed that all degraded proteins should leave the cells. However, a more realistic situation is the presence of recycling, i. e., when only some of the monomers appearing as a result of protein degradation are removed, while the other ones stay in the cells and are used as precursor metabolites again. Although protein recycling has never been explicitly quantified in bacteria to our knowledge, it has been observed for several hours in Escherichia coli strains with specific amino acid requirements [18].

In this paper, we extend the dynamic models of $[8,17]$ by including both degradation and recycling. Important analytical and computational results for the corresponding growth maximization problem are also obtained. In particular, we provide a detailed formulation and justification for a numerical method to construct the optimal feedback resource allocation (control) strategy. The feedback form is useful there not only due to the aim of combining all possible initial states together but also because it can help to identify, for instance, the connection with the abovementioned ppGpp-based strategy in Escherichia coli [7,8]. Furthermore, such works as [19-22] give us a biological motivation to study how increases of temperature (which intensify protein degradation) affect our model and the optimal feedback control law. A relevant question is whether the bacterial growth maximization strategy is highly sensitive to temperature in certain ranges or not.

The paper is organized as follows. Section 2 contains the statement of our dynamic optimization problem with a comprehensive theoretical discussion. After establishing some auxiliary results in Section 3, we provide the steady-state analysis in Section 4 and prove the existence of optimal openloop control strategies in Section 5. Based on a detailed analytical investigation of the problem by Pontryagin's maximum principle [23] in Section 6, we develop an approach to approximate the switching curve of the optimal feedback control law in Section 7. Note that the constructed field of extremal state trajectories consists of chattering arcs and one steady-state singular arc [24,25]. Section 8 presents the results of numerical simulations, including the dependence of the optimal control synthesis on temperature. Concluding remarks are given in Section 9. There are also two appendices. The first one describes a convenient technique for transforming our model in case of a 


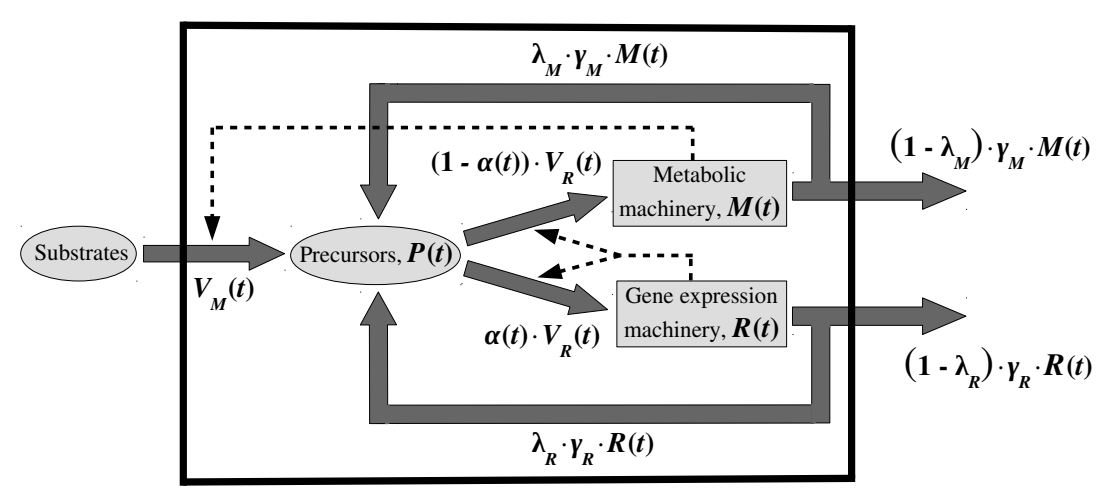

Figure 1. Scheme of the model. Metabolic machinery transforms external substrates (nutrients) into precursors. Gene expression machinery converts the precursors into macromolecules (proteins), which are involved either in metabolism or in gene expression itself. Macromolecular degradation also takes place. The proteins degrade back into precursors, and some of the latter are removed from the cells, while the other ones are recycled and used as internal construction units again.

temperature change, and the second one tests the numerical method of Section 7 on classical Fuller problem.

\section{PROBLEM STATEMENT}

\subsection{Dynamical system}

Consider a population of self-replicating prokaryotic cells (such as, for example, Escherichia coli enterobacteria). Let $P, M, R$ [g] be the total masses of precursor metabolites (amino acids), metabolic machinery (enzymes involved in nutrient uptake and conversion into precursors), and gene expression machinery (polymerase, ribosomes), respectively. A scheme of the model (with the related mass fluxes and catalytic effects) is shown in Fig. 1. Metabolic machinery transforms external substrates (nutrients) into precursors. Gene expression machinery converts the precursors into macromolecules (proteins), which are involved either in metabolism or in gene expression itself. Macromolecular degradation also takes place. The proteins degrade back into precursors, and some of the latter are removed from the cells, while the other ones are recycled and used as internal construction units again.

Thus, from the mass balance, we arrive at the controlled system of ordinary differential equations

$$
\left\{\begin{array}{l}
\frac{d P(t)}{d t}=V_{M}(t)-V_{R}(t)+\lambda_{M} \gamma_{M} M(t)+\lambda_{R} \gamma_{R} R(t), \\
\frac{d M(t)}{d t}=(1-\alpha(t)) V_{R}(t)-\gamma_{M} M(t), \\
\frac{d R(t)}{d t}=\alpha(t) V_{R}(t)-\gamma_{R} R(t), \\
\alpha_{\min } \leqslant \alpha(t) \leqslant \alpha_{\max }, \quad t \in[0, T],
\end{array}\right.
$$

where the following notations are adopted: 
- $t[\mathrm{~h}]$ is the time variable, $T>0[\mathrm{~h}]$ is a time horizon;

- $V_{M}=V_{M}(t)\left[\mathrm{g} \cdot \mathrm{h}^{-1}\right]$ and $V_{R}=V_{R}(t)\left[\mathrm{g} \cdot \mathrm{h}^{-1}\right]$ are the rates at which the precursors are built by metabolism and utilized for gene expression, respectively;

- $\gamma_{M} \geqslant 0\left[\mathrm{~h}^{-1}\right]$ and $\gamma_{R} \geqslant 0\left[\mathrm{~h}^{-1}\right]$ are the degradation rates for metabolic and gene expression machineries, respectively;

- $\lambda_{M} \in[0,1]$ and $\lambda_{R} \in[0,1]$ are dimensionless recycling parameters, so that $\lambda_{M} \cdot \gamma_{M}$ and $\lambda_{R} \cdot \gamma_{R}$ are the resulting recycling coefficients;

- $\alpha=\alpha(t)$ is a dimensionless resource allocation function (control strategy) such that, at any time instant $t \in[0, T], \alpha(t)$ is the proportion of the precursor mass used for supporting gene expression, and $1-\alpha(t)$ is the proportion for metabolism;

- $\alpha_{\min } \in[0,1)$ and $\alpha_{\max } \in\left(\alpha_{\min }, 1\right]$ are constants specifying lower and upper constraints on admissible resource allocation functions.

It is necessary to emphasize some general simplifications on which the presented model relies. First, only two classes of macromolecules (with the total masses $M$ and $R$ ) are considered. In line with [16], we in fact suppose that the core sector of macromolecules for cell maintenance [16], sensing and regulatory mechanisms [26, 27], proteolysis [28], etc., is fixed and can thereby be neglected in our resource allocation problem. Moreover, operating with $P, M, R$ as with total masses becomes reasonable only under the assumption that individual cells in the studied population are similar to each other.

The system (1) extends the dynamic models of $[8,17]$ by including degradation rates and recycling terms together (the model of [8] contains no degradation, while the model of [17] considers degradation without recycling).

As in $[8,17]$, introduce the following new notations:

- all cells in the considered population are supposed to have the same constant cytoplasmic density, and $\beta>0\left[\mathrm{~L} \cdot \mathrm{g}^{-1}\right]$ is its inverse;

- the quantity

$$
\mathcal{V}(t) \stackrel{\text { def }}{=} \beta \cdot(M(t)+R(t)) \quad[\mathrm{L}]
$$

is interpreted as the structural volume of the cell population (in the sense of the macromolecules constituting metabolic and gene expression machineries, so that monomer precursors are not included here);

- the quantities

$$
\begin{gathered}
p(t) \stackrel{\text { def }}{=} \frac{P(t)}{\mathcal{V}(t)} \quad\left[\mathrm{g} \cdot \mathrm{L}^{-1}\right], \quad r(t) \stackrel{\text { def }}{=} \frac{R(t)}{\mathcal{V}(t)} \quad\left[\mathrm{g} \cdot \mathrm{L}^{-1}\right], \\
m(t) \stackrel{\text { def }}{=} \frac{M(t)}{\mathcal{V}(t)}=\frac{1}{\beta}-r(t) \\
v_{M}(t) \stackrel{\left.\mathrm{g} \cdot \mathrm{L}^{-1}\right],}{=} \frac{V_{M}(t)}{\mathcal{V}(t)} \quad\left[\mathrm{g} \cdot \mathrm{L}^{-1} \cdot \mathrm{h}^{-1}\right], \quad v_{R}(t) \stackrel{\text { def }}{=} \frac{V_{R}(t)}{\mathcal{V}(t)} \quad\left[\mathrm{g} \cdot \mathrm{L}^{-1} \cdot \mathrm{h}^{-1}\right]
\end{gathered}
$$

are intracellular analogues for $P(t), R(t), M(t), V_{M}(t), V_{R}(t)$, respectively; 
- the growth rate of the self-replicating system is defined as the relative increase in the volume (2), i.e., as

$$
\begin{aligned}
\mu(t) \stackrel{\text { def }}{=} \frac{1}{\mathcal{V}(t)} \frac{d \mathcal{V}(t)}{d t} & =\beta\left(v_{R}(t)-\gamma_{M} m(t)-\gamma_{R} r(t)\right) \\
& =\beta\left(v_{R}(t)+\left(\gamma_{M}-\gamma_{R}\right) r(t)-\frac{\gamma_{M}}{\beta}\right) .
\end{aligned}
$$

Then the system (1) takes the form

$$
\left\{\begin{aligned}
& \frac{d p(t)}{d t}= v_{M}(t)-v_{R}(t)+\frac{\lambda_{M} \gamma_{M}}{\beta}-\left(\lambda_{M} \gamma_{M}-\lambda_{R} \gamma_{R}\right) r(t) \\
&-\beta p(t)\left(v_{R}(t)+\left(\gamma_{M}-\gamma_{R}\right) r(t)-\frac{\gamma_{M}}{\beta}\right) \\
& \frac{d r(t)}{d t}= \alpha(t) v_{R}(t)-\gamma_{R} r(t) \\
&-\beta r(t)\left(v_{R}(t)+\left(\gamma_{M}-\gamma_{R}\right) r(t)-\frac{\gamma_{M}}{\beta}\right) \\
& \alpha_{\min } \leqslant \alpha(t) \leqslant \alpha_{\max }, \quad t \in[0, T]
\end{aligned}\right.
$$

(the number of differential equations is decreased from 3 to 2 due to the third representation in (3)).

According to $[8,17]$, the rate of precursor synthesis is catalyzed by metabolic machinery and determined as the linear function

$$
v_{M}(t)=e_{M} \cdot m(t)=e_{M} \cdot\left(\frac{1}{\beta}-r(t)\right),
$$

while the rate of protein synthesis is catalyzed by gene expression machinery and specified through the classical Michaelis-Menten law:

$$
v_{R}(t)=k_{R} \cdot r(t) \cdot \frac{p(t)}{K_{R}+p(t)} .
$$

Here $e_{M}>0\left[\mathrm{~h}^{-1}\right]$ is the constant environmental input reflecting the concentration and quality of external substrates, whereas $k_{R}>0\left[\mathrm{~h}^{-1}\right]$ and $K_{R}>0\left[\mathrm{~g} \cdot \mathrm{L}^{-1}\right]$ are respectively the rate and halfsaturation constants for protein synthesis. Note that (6) is a particular form of the Michaelis-Menten law for the case when the environmental input

$$
e_{M}(t) \stackrel{\text { def }}{=} k_{M} \cdot \frac{s(t)}{K_{M}+s(t)}
$$

almost does not change and can therefore be treated as a constant parameter. In (8), $k_{M}>0\left[\mathrm{~h}^{-1}\right]$ and $K_{M}>0\left[\mathrm{~g} \cdot \mathrm{L}^{-1}\right]$ are respectively the rate and half-saturation constants for metabolism, while $s(t)$ is the nutrient concentration in the medium. It is reasonable to suppose $e_{M}=$ const if $s(t) \approx$ const or if $s(t) \gg K_{M}$ (the latter means that the substrates are available in excess). 
Now let us rescale the model in order to simplify its mathematical investigation. By using the new dimensionless variables

$$
\hat{t} \stackrel{\text { def }}{=} k_{R} \cdot t, \quad \hat{p}(\hat{t}) \stackrel{\text { def }}{=} \beta \cdot p(t), \quad \hat{r}(\hat{t}) \stackrel{\text { def }}{=} \beta \cdot r(t)
$$

and constants

$$
\begin{gathered}
\hat{T} \stackrel{\text { def }}{=} k_{R} \cdot T, \quad E_{M} \stackrel{\text { def }}{=} \frac{e_{M}}{k_{R}}, \quad K \stackrel{\text { def }}{=} \beta \cdot K_{R}, \\
\Gamma_{M} \stackrel{\text { def }}{=} \frac{\gamma_{M}}{k_{R}}, \quad \Gamma_{R} \stackrel{\text { def }}{=} \frac{\gamma_{R}}{k_{R}},
\end{gathered}
$$

the system (5) can be transformed into

$$
\left\{\begin{aligned}
& \frac{d \hat{p}(\hat{t})}{d \hat{t}}=(1-\hat{r}(\hat{t})) E_{M}-\frac{\hat{p}(\hat{t}) \hat{r}(\hat{t})}{K+\hat{p}(\hat{t})} \\
&+\lambda_{M} \Gamma_{M}-\left(\lambda_{M} \Gamma_{M}-\lambda_{R} \Gamma_{R}\right) \hat{r}(\hat{t}) \\
&-\hat{p}(\hat{t})\left(\frac{\hat{p}(\hat{t}) \hat{r}(\hat{t})}{K+\hat{p}(\hat{t})}+\left(\Gamma_{M}-\Gamma_{R}\right) \hat{r}(\hat{t})-\Gamma_{M}\right), \\
& \frac{d \hat{r}(\hat{t})}{d \hat{t}}= \alpha(\hat{t}) \frac{\hat{p}(\hat{t}) \hat{r}(\hat{t})}{K+\hat{p}(\hat{t})}-\Gamma_{R} \hat{r}(\hat{t}) \\
&-\hat{r}(\hat{t})\left(\frac{\hat{p}(\hat{t}) \hat{r}(\hat{t})}{K+\hat{p}(\hat{t})}+\left(\Gamma_{M}-\Gamma_{R}\right) \hat{r}(\hat{t})-\Gamma_{M}\right), \\
& \alpha_{\min } \leqslant \alpha(\hat{t}) \leqslant \alpha_{\max }, \quad \hat{t} \in[0, \hat{T}]
\end{aligned}\right.
$$

The initial conditions are fixed as

$$
\hat{p}(0)=\hat{p}_{0}, \quad \hat{r}(0)=\hat{r}_{0}
$$

\subsection{Optimal control problem}

In compliance with $[8,13-15,17]$, we adopt the hypothesis that regulation mechanisms in the cells are aimed at maximizing the accumulation of structural biomass under current environmental conditions. For our model, this leads to the criterion

$$
\begin{gathered}
\int_{0}^{T} \mu(t) d t=\int_{0}^{\hat{T}} \hat{\mu}(\hat{t}) d \hat{t} \longrightarrow \max _{\alpha(\cdot)}, \\
\hat{\mu}(\hat{t}) \stackrel{\text { def }}{=} \frac{\mu(t)}{k_{R}}=\frac{\hat{p}(\hat{t}) \hat{r}(\hat{t})}{K+\hat{p}(\hat{t})}+\left(\Gamma_{M}-\Gamma_{R}\right) \hat{r}(\hat{t})-\Gamma_{M} .
\end{gathered}
$$

Here the maximum is searched over all admissible open-loop control strategies, which are measurable functions $\alpha:[0, \hat{T}] \rightarrow\left[\alpha_{\min }, \alpha_{\max }\right]$. 
For our studies, it is reasonable to assume that changes in the environment (i. e., in nutrient availability) are possible only in the form of instantaneous shifts [8, 17, 29]. For example, Escherichia coli bacteria cycle between various habitats such as mammalian intestinal systems, water bodies, soils, sediments, etc. We impose the condition that intermediate environmental fluctuations between the shifts are negligible.

Any nutrient downshift or upshift leads to a new value of the environmental input $e_{M}$ and thereby changes the optimal resource allocation problem (11)-(13). In the current work, we model bacterial adaptation mechanisms on the time interval between two arbitrary consecutive shifts of the environment. Hence, $e_{M}$ is taken as a constant parameter in the problem (11)-(13).

Note that the growth maximization criterion in the problem of [8] was infinite-horizon and understood in the overtaking optimality sense [30]. Analytical reasonings for this problem were based on the implicit assumption that the optimal state trajectories should enter a specific rest point (with the maximum growth rate) and stay there all the remaining infinite time. However, it was not proved that the overtaking optimal control strategies existed and generated such trajectories. Besides, the infinite-horizon case could not be treated numerically, while the approximate optimal control functions for the problem with a fixed finite horizon and a free terminal state acted so that the mentioned rest point was left a little time before the final instant. By referring to the general turnpike theory of [31], the hypothesis on absence of such exiting subarcs in the overtaking optimal processes was proposed (and these subarcs were removed from the illustrations), but rigorous verification remained an open task there.

Thus, we need a somewhat different statement of our dynamic optimization problem in order to allow wider mathematical justification, while preserving a reasonable biological interpretation.

First, one can think of following the economic modeling framework of [32] and thereby including the exponential decaying factor $e^{-\nu t}$ in the maximized improper integral of the growth rate. Such a discounted functional was also considered in [12]. However, its biological meaning and a method to estimate a suitable value of the parameter $\nu$ from real data are not totally clear.

Let us adopt the approach of [17], which is to take a sufficiently large finite time horizon $\hat{T}$ and to impose the terminal condition

$$
(\hat{p}(\hat{T}), \hat{r}(\hat{T}))=\left(\hat{p}_{\mathrm{opt}}^{*}, \hat{r}_{\mathrm{opt}}^{*}\right) \quad(\hat{T} \in(0,+\infty) \text { is fixed }),
$$

where $\left(\hat{p}_{\text {opt }}^{*}, \hat{r}_{\text {opt }}^{*}\right)$ is the steady state with the maximum growth rate. The related steady-state analysis and, in particular, steady-state optimization will be provided in Section 4.

For the dynamic optimization problem (11)-(14) under some natural assumptions, it becomes possible to prove existence of optimal open-loop control strategies if $\hat{T}$ is large enough. This will be done in Section 5, and a practical recommendation for choosing suitable time horizons will also be given there. In Section 6, we will establish that the optimal state trajectories reach the steady state $\left(\hat{p}_{\text {opt }}^{*}, \hat{r}_{\text {opt }}^{*}\right)$ by chattering [24,25]. Section 7 will develop a numerical method to approximate the corresponding switching curve in the state space. One more significant argument in favor of our problem statement with the terminal constraint (14) is that this method in principle will not depend on a particular time horizon, i. e., we will obtain the same results when applying it to the related infinite-horizon problem in the overtaking optimality sense (under the a priori assumption of entering $\left(\hat{p}_{\mathrm{opt}}^{*}, \hat{r}_{\mathrm{opt}}^{*}\right)$ by the overtaking optimal state trajectories). 


\subsection{Effect of temperature}

From both theoretical and practical point of view, it is important to understand influence of temperature on biochemical systems [19-22,33]. The works $[19,20]$ contained experimental studies of the temperature effect on the growth and degradation for Escherichia coli. The experiments of [19] showed the reduction of the growth rate from nearly its maximum at $37^{\circ} \mathrm{C}$ to about $1 \%$ of that at $45^{\circ} \mathrm{C}$, which was called forth by the difference in sensitivity to temperature changes between the protein synthesis and degradation. An approach to account for this property within the framework of the model (11) is described in Appendix A. The key aspect is to use Arrhenius law [33] with different activation energies for the protein synthesis and degradation. Finally, the form of the dynamical system remains similar, but the time variable $\hat{t}$ and parameters $\Gamma_{M}, \Gamma_{R}$ change so that the influence of the degradation rates increases with the increase of temperature. One more objective of this work is to investigate how the optimal feedback control law for the problem (11)-(13) depends on temperature. The results of the related numerical simulations will be presented in Section 8 .

\section{PRELIMINARY CONSIDERATIONS}

Before proceeding to the primary investigation, let us establish some auxiliary results. Several introductory assumptions will also be formulated in this section. Further assumptions will be given in the subsequent sections when needed.

First, recall the a priori conditions on the model parameters.

Assumption 3.1

$\gamma_{M} \geqslant 0, \gamma_{R} \geqslant 0,0 \leqslant \lambda_{M} \leqslant 1,0 \leqslant \lambda_{R} \leqslant 1,0 \leqslant \alpha_{\min }<\alpha_{\max } \leqslant 1, \beta>0, e_{M}>0, k_{R}>0$, $K_{R}>0$.

Assumption 3.1 and the definitions (10) trivially imply $E_{M}>0, K>0, \Gamma_{M} \geqslant 0, \Gamma_{R} \geqslant 0$.

It is convenient to specify a suitable strongly invariant region [34, Chapter $4, \S 3]$ for our controlled system.

Proposition 3.1

Let Assumption 3.1 hold. For the controlled system (11), the set

$$
G \stackrel{\text { def }}{=}\{(\hat{p}, \hat{r}): \hat{p}>0,0<\hat{r}<1\}
$$

is a strongly invariant domain in the state space, i.e., admissible open-loop control strategies generate such state trajectories that cannot leave $G$ if the corresponding initial states lie in $G$.

Proof

Suppose that $\hat{p}_{0} \geqslant 0$ and $0 \leqslant \hat{r}_{0} \leqslant 1$. Consider an arbitrary measurable control function $\alpha: I \rightarrow$ $\left[\alpha_{\min }, \alpha_{\max }\right]$ defined on some connected time interval $I \subseteq[0,+\infty)$ with $0 \in I$. Let $(\hat{p}(\cdot), \hat{r}(\cdot))$ be a solution to (11),(12) on $I$ for the chosen $\alpha(\cdot)$. If $\hat{r}_{0}=0$, then $d \hat{r}(\hat{t}) / d \hat{t} \equiv 0$ and $\hat{r}(\hat{t}) \equiv 0$ on $I$. For $\hat{r}_{0}>0$, we have $\hat{r}(\hat{t})>0$ everywhere on $I$. It suffices to verify the following two implications:

1) if $\hat{p}_{0}>0$ and $0 \leqslant \hat{r}(\hat{t}) \leqslant 1$ everywhere on $I$, then $\hat{p}(\hat{t})>0$ everywhere on $I$;

2) if $0<\hat{r}_{0}<1$ and $\hat{p}(\hat{t}) \geqslant 0$ everywhere on $I$, then $\hat{r}(\hat{t})<1$ everywhere on $I$. 
Let us prove the implication 1 . When $\hat{p}\left(\hat{t}^{\prime}\right)=0$ at some $\hat{t}^{\prime} \in I$, the derivative $d \hat{p}\left(\hat{t}^{\prime}\right) / d \hat{t}$ is zero for $1-\hat{r}\left(\hat{t}^{\prime}\right)=\lambda_{R} \Gamma_{R}=0$ and positive in the following cases: a) $\hat{r}\left(\hat{t}^{\prime}\right)=1, \lambda_{R} \Gamma_{R}>0$; b) $0 \leqslant \hat{r}\left(\hat{t}^{\prime}\right)<1$. Therefore, if $\hat{p}_{0}>0$ and $0 \leqslant \hat{r}(\hat{t}) \leqslant 1$ everywhere on $I$, then we may have $\hat{p}\left(\hat{t}^{\prime}\right)=0$ at some $\hat{t}^{\prime} \in I$ only for $1-\hat{r}\left(\hat{t}^{\prime}\right)=\lambda_{R} \Gamma_{R}=0$. However, for $\lambda_{R} \Gamma_{R}=0$, the point $(\hat{p}, \hat{r})=(0,1)$ is a steady state of the system regardless of a control $\alpha(\cdot)$. Thus, the implication 1 indeed holds.

Now let us establish the implication 2. Assume that $0<\hat{r}_{0}<1$ and $\hat{p}(\hat{t}) \geqslant 0$ everywhere on $I$. From the second equation in (11), we get

$$
\begin{aligned}
& \frac{d \hat{r}(\hat{t})}{d \hat{t}} \leqslant(1-\hat{r}(\hat{t})) \frac{\hat{p}(\hat{t}) \hat{r}(\hat{t})}{K+\hat{p}(\hat{t})}+\left(\Gamma_{M}-\Gamma_{R}\right) \hat{r}(\hat{t})(1-\hat{r}(\hat{t})), \\
& \frac{d}{d \hat{t}}(1-\hat{r}(\hat{t})) \geqslant-\hat{r}(\hat{t})(1-\hat{r}(\hat{t}))\left(\frac{\hat{p}(\hat{t})}{K+\hat{p}(\hat{t})}+\Gamma_{M}-\Gamma_{R}\right)
\end{aligned}
$$

for all $\hat{t} \in I$. Let $\rho: I \rightarrow \mathbb{R}$ be the solution to the Cauchy problem

$$
\left\{\begin{array}{l}
\frac{d \rho(\hat{t})}{d \hat{t}}=-(1-\rho(\hat{t})) \rho(\hat{t})\left(\frac{\hat{p}(\hat{t})}{K+\hat{p}(\hat{t})}+\Gamma_{M}-\Gamma_{R}\right), \quad \hat{t} \in I, \\
\rho(0)=1-\hat{r}_{0} .
\end{array}\right.
$$

Since $\rho(0)>0$, then $\rho(\cdot)$ is positive everywhere on $I$. According to the well-known general comparison result [35, Theorem 1.10.2], we obtain $1-\hat{r}(\hat{t}) \geqslant \rho(\hat{t})>0$ and, consequently, $\hat{r}(\hat{t})<1$ for all $\hat{t} \in I$. This completes the proof.

Besides, the first equation in (11) implies that

$$
\begin{aligned}
\frac{d \hat{p}(\hat{t})}{d \hat{t}}< & \max _{\eta \in[0,1]}\left\{\left(E_{M}+\lambda_{M} \Gamma_{M}\right)(1-\eta)+\lambda_{R} \Gamma_{R} \eta\right\} \\
& +\hat{p}(\hat{t}) \cdot \max _{\eta \in[0,1]}\left\{\Gamma_{M}-\left(\Gamma_{M}-\Gamma_{R}\right) \eta\right\}
\end{aligned}
$$

when $(\hat{p}(\hat{t}), \hat{r}(\hat{t})) \in G$. For any $q_{0} \in \mathbb{R}$, the Cauchy problem

$$
\left\{\begin{aligned}
\begin{array}{rl}
\frac{d q(\hat{t})}{d \hat{t}}= & \max _{\eta \in[0,1]}\left\{\left(E_{M}+\lambda_{M} \Gamma_{M}\right)(1-\eta)+\lambda_{R} \Gamma_{R} \eta\right\} \\
& +q(\hat{t}) \cdot \max _{\eta \in[0,1]}\left\{\Gamma_{M}-\left(\Gamma_{M}-\Gamma_{R}\right) \eta\right\}, \quad \hat{t} \geqslant 0
\end{array} \\
\\
q(0)=q_{0},
\end{aligned}\right.
$$

obviously has a unique solution $q\left(\cdot ; q_{0}\right)$ (which can serve as a dynamic upper estimate for $\hat{p}(\cdot)$ if $q_{0}=\hat{p}_{0}$ ). Then, with the help of the comparison result [35, Theorem 1.10.2] and Proposition 3.1, we arrive at the following corollary.

\section{Corollary 3.1}

For any measurable function $\alpha:[0,+\infty) \rightarrow\left[\alpha_{\min }, \alpha_{\max }\right]$ and any initial state $\left(\hat{p}_{0}, \hat{r}_{0}\right) \in G$, there 
exists a unique solution to (11),(12) on the time interval $[0,+\infty)$, and, moreover,

$$
\begin{gathered}
(\hat{p}(\hat{t}), \hat{r}(\hat{t})) \in G \quad \forall \hat{t} \geqslant 0, \\
\hat{p}(\hat{t}) \leqslant q\left(\hat{t} ; \hat{p}_{0}\right) \leqslant \max _{\xi \in[0, \hat{T}]} q\left(\xi ; \hat{p}_{0}\right) \quad \forall \hat{t} \in[0, \hat{T}] .
\end{gathered}
$$

Let us impose the next condition in order to consider only such state trajectories of (11) that lie in $G$.

Assumption 3.2

$\hat{p}_{0}>0,0<\hat{r}_{0}<1$.

It is also natural to suppose that the macromolecules used in metabolism are less stable than those for gene expression $[17,18,36-38]$.

\section{Assumption 3.3}

$\Gamma_{M} \geqslant \Gamma_{R}$.

The next result will be used in Section 5. It gives a sufficient condition for boundedness of solutions to $(11),(12)$ on the infinite time interval $[0,+\infty)$.

\section{Proposition 3.2}

Let Assumptions 3.1-3.3 hold, and let $\alpha:[0,+\infty) \rightarrow\left[\alpha_{\min }, \alpha_{\max }\right]$ be a measurable function such that

$$
\alpha(\hat{t})>\Gamma_{R}+\varepsilon \quad \forall \hat{t} \geqslant 0,
$$

where $\varepsilon>0$ is some constant. Then the corresponding solution $(\hat{p}(\cdot), \hat{r}(\cdot))$ to $(11),(12)$ on the time interval $[0,+\infty)$ is bounded.

Proof

Due to Corollary 3.1, it suffices to verify existence of such a finite uniform upper bound for $\hat{p}(\cdot)$ that may depend only on the model parameters and initial state $\left(\hat{p}_{0}, \hat{r}_{0}\right)$.

The second equation in (11) can be rewritten as

$$
\frac{d \hat{r}(\hat{t})}{d \hat{t}}=\hat{r}(\hat{t})\left(\frac{\hat{p}(\hat{t})}{K+\hat{p}(\hat{t})}+\Gamma_{M}-\Gamma_{R}\right)\left(\hat{r}_{1}^{*}(\hat{p}(\hat{t}), \alpha(\hat{t}))-\hat{r}(\hat{t})\right),
$$

where

$$
\hat{r}_{1}^{*}(\hat{p}, \alpha) \stackrel{\text { def }}{=} \frac{\alpha \cdot \frac{\hat{p}}{K+\hat{p}}+\Gamma_{M}-\Gamma_{R}}{\frac{\hat{p}}{K+\hat{p}}+\Gamma_{M}-\Gamma_{R}} \geqslant \alpha \quad \forall \hat{p}>0 \quad \forall \alpha \in\left[\alpha_{\min }, \alpha_{\max }\right] .
$$

This yields

$$
\hat{r}(\hat{t}) \geqslant \hat{r}_{\text {low }} \stackrel{\text { def }}{=} \min \left(\Gamma_{R}+\varepsilon, \hat{r}_{0}\right)>0 \quad \forall \hat{t} \geqslant 0
$$

by virtue of (16). 
Now, by using both equations in (11), we get

$$
\begin{aligned}
\frac{d}{d \hat{t}}\left(\frac{\hat{p}(\hat{t})}{\hat{r}(\hat{t})}\right)= & \left(E_{M}+\lambda_{M} \Gamma_{M}\right)\left(\frac{1}{\hat{r}(\hat{t})}-1\right)-\frac{\hat{p}(\hat{t})}{K+\hat{p}(\hat{t})}+\lambda_{R} \Gamma_{R} \\
& -\frac{\hat{p}(\hat{t})}{\hat{r}(\hat{t})}\left(\alpha(\hat{t}) \cdot \frac{\hat{p}(\hat{t})}{K+\hat{p}(\hat{t})}-\Gamma_{R}\right) .
\end{aligned}
$$

Note that

$$
\frac{1}{\hat{r}(\hat{t})} \leqslant \frac{1}{\hat{r}_{\text {low }}}, \quad \hat{p}(\hat{t})<\frac{\hat{p}(\hat{t})}{\hat{r}(\hat{t})} \leqslant \frac{\hat{p}(\hat{t})}{\hat{r}_{\text {low }}} \quad \forall \hat{t} \geqslant 0
$$

due to (17), and, furthermore, $\hat{p} /(K+\hat{p}) \underset{\hat{p} \rightarrow+\infty}{\longrightarrow} 1$. Hence, the right-hand side of (18) is negative when $\hat{p}(\hat{t})$ is sufficiently large. With the help of (17)-(19), one can directly obtain a suitable finite upper bound for $\hat{p}(\cdot)$ depending only on the model parameters and initial state.

\section{STEADY-STATE ANALYSIS}

Let us find a steady state $\left(\hat{p}_{\text {opt }}^{*}, \hat{r}_{\text {opt }}^{*}\right) \in G$ at which the growth rate (4) takes the maximum value. Some new conditions on the model parameters need to be imposed.

\section{Assumption 4.1}

The following inequalities hold:

$$
\begin{gathered}
E_{M}+\left(\lambda_{M}-\lambda_{R}\right) \Gamma_{M}>0 \\
\left(1-\Gamma_{R}\right) E_{M}-\Gamma_{M}\left(1-\lambda_{M}+\left(\lambda_{M}-\lambda_{R}\right) \Gamma_{R}\right)>0 .
\end{gathered}
$$

Assumption 4.1 and also subsequent Assumptions 4.4, 5.1 are not just technical. In particular, they require the environmental input to be high enough and the degradation rates to be low enough, so that the bacterial growth could be maintained at an acceptable level.

For any steady state $\left(\hat{p}^{*}, \hat{r}^{*}\right) \in G$ of the system (11) with the corresponding control $\alpha^{*}$, we have

$$
\hat{r}^{*}=\hat{r}^{*}\left(\hat{p}^{*}\right)=\frac{E_{M}+\lambda_{M} \Gamma_{M}+\Gamma_{M} \hat{p}^{*}}{E_{M}+\lambda_{M} \Gamma_{M}-\lambda_{R} \Gamma_{R}+\frac{\hat{p}^{*}\left(1+\hat{p}^{*}\right)}{K+\hat{p}^{*}}+\left(\Gamma_{M}-\Gamma_{R}\right) \hat{p}^{*}}
$$

(here the denominator is positive by virtue of Assumption 3.3 and the first inequality in Assumption 4.1, which yield $\left.E_{M}+\lambda_{M} \Gamma_{M}-\lambda_{R} \Gamma_{R} \geqslant E_{M}+\left(\lambda_{M}-\lambda_{R}\right) \Gamma_{M}>0\right)$ and

$$
\alpha^{*}=\alpha^{*}\left(\hat{p}^{*}\right)=\hat{r}^{*}\left(\hat{p}^{*}\right)-\left(\Gamma_{M}-\Gamma_{R}\right) \frac{K+\hat{p}^{*}}{\hat{p}^{*}}\left(1-\hat{r}^{*}\left(\hat{p}^{*}\right)\right)
$$

(if the latter expression belongs to $\left[\alpha_{\min }, \alpha_{\max }\right]$ ). According to (13), our aim is to maximize

$$
\hat{\mu}^{*}=\hat{\mu}^{*}\left(\hat{p}^{*}\right)=\frac{\hat{p}^{*} \hat{r}^{*}\left(\hat{p}^{*}\right)}{K+\hat{p}^{*}}+\left(\Gamma_{M}-\Gamma_{R}\right) \hat{r}^{*}\left(\hat{p}^{*}\right)-\Gamma_{M}
$$

over all $\hat{p}^{*}>0$. 
One can directly verify that

$$
\begin{aligned}
\hat{\mu}^{*}\left(\hat{p}^{*}\right)= & \left(\left(1+\Gamma_{M}-\Gamma_{R}\right) \cdot\left(\hat{p}^{*}\right)^{2}+\left(E_{M}+\lambda_{M} \Gamma_{M}-\lambda_{R} \Gamma_{R}+1+\left(\Gamma_{M}-\Gamma_{R}\right) K\right) \cdot \hat{p}^{*}\right. \\
& \left.+K\left(E_{M}+\lambda_{M} \Gamma_{M}-\lambda_{R} \Gamma_{R}\right)\right)^{-1} \cdot \\
& \quad\left(\left(\left(1-\Gamma_{R}\right) E_{M}-\Gamma_{M}\left(1-\lambda_{M}+\left(\lambda_{M}-\lambda_{R}\right) \Gamma_{R}\right)\right) \cdot \hat{p}^{*}\right. \\
& \left.\quad-\Gamma_{R} K\left(E_{M}+\left(\lambda_{M}-\lambda_{R}\right) \Gamma_{M}\right)\right)
\end{aligned}
$$

and, consequently,

$$
\begin{array}{r}
\frac{d \hat{\mu}^{*}\left(\hat{p}^{*}\right)}{d \hat{p}^{*}} \cdot\left(\left(1+\Gamma_{M}-\Gamma_{R}\right) \cdot\left(\hat{p}^{*}\right)^{2}+\left(E_{M}+\lambda_{M} \Gamma_{M}-\lambda_{R} \Gamma_{R}+1+\left(\Gamma_{M}-\Gamma_{R}\right) K\right) \cdot \hat{p}^{*}\right. \\
\left.+K\left(E_{M}+\lambda_{M} \Gamma_{M}-\lambda_{R} \Gamma_{R}\right)\right)^{2}=-a \cdot\left(\hat{p}^{*}\right)^{2}+b \cdot \hat{p}^{*}+c
\end{array}
$$

where

$$
\begin{gathered}
a \stackrel{\text { def }}{=}\left(\left(1-\Gamma_{R}\right) E_{M}-\Gamma_{M}\left(1-\lambda_{M}+\left(\lambda_{M}-\lambda_{R}\right) \Gamma_{R}\right)\right) \cdot\left(1+\Gamma_{M}-\Gamma_{R}\right), \\
b \stackrel{\text { def }}{=} 2 \Gamma_{R} K\left(E_{M}+\left(\lambda_{M}-\lambda_{R}\right) \Gamma_{M}\right) \cdot\left(1+\Gamma_{M}-\Gamma_{R}\right), \\
c \stackrel{\text { def }}{=} K \cdot\left(\left(\left(1-\Gamma_{R}\right) E_{M}-\Gamma_{M}\left(1-\lambda_{M}+\left(\lambda_{M}-\lambda_{R}\right) \Gamma_{R}\right)\right) \cdot\right. \\
\cdot\left(E_{M}+\lambda_{M} \Gamma_{M}-\lambda_{R} \Gamma_{R}\right)+\Gamma_{R}\left(E_{M}+\left(\lambda_{M}-\lambda_{R}\right) \Gamma_{M}\right) . \\
\left.\cdot\left(E_{M}+\lambda_{M} \Gamma_{M}-\lambda_{R} \Gamma_{R}+1+\left(\Gamma_{M}-\Gamma_{R}\right) K\right)\right) .
\end{gathered}
$$

Assumptions 3.3 and 4.1 imply positivity of the constants $a, b, c$. Then

$$
\hat{p}_{\text {opt }}^{*} \stackrel{\text { def }}{=} \frac{b+\sqrt{b^{2}+4 a c}}{2 a}
$$

is a unique positive root of the equation $d \hat{\mu}^{*}\left(\hat{p}^{*}\right) / d \hat{p}^{*}=0$. This is indeed a unique maximum point of the function (22) on the interval $[0,+\infty)$, since

$$
\frac{d \hat{\mu}^{*}\left(\hat{p}^{*}\right)}{d \hat{p}^{*}}>0 \quad \forall \hat{p}^{*} \in\left[0, \hat{p}_{\mathrm{opt}}^{*}\right), \quad \frac{d \hat{\mu}^{*}\left(\hat{p}^{*}\right)}{d \hat{p}^{*}}<0 \quad \forall \hat{p}^{*}>\hat{p}_{\mathrm{opt}}^{*} .
$$

The next assumption is required in order to guarantee that the optimal steady-state regime is feasible and related to an intermediate configuration of resource allocation.

\section{Assumption 4.2}

$\hat{r}_{\mathrm{opt}}^{*} \stackrel{\text { def }}{=} \hat{r}^{*}\left(\hat{p}_{\mathrm{opt}}^{*}\right) \in(0,1), \alpha_{\mathrm{opt}}^{*} \stackrel{\text { def }}{=} \alpha^{*}\left(\hat{p}_{\mathrm{opt}}^{*}\right) \in\left(\alpha_{\min }, \alpha_{\mathrm{max}}\right)$.

Similarly, denote $\hat{\mu}_{\mathrm{opt}}^{*} \stackrel{\text { def }}{=} \hat{\mu}^{*}\left(\hat{p}_{\mathrm{opt}}^{*}\right)$. 
Now consider the Jacobian matrix of the system (11):

$$
\begin{gathered}
D(\hat{p}, \hat{r} ; \alpha)=\left(\begin{array}{cc}
d_{11}(\hat{p}, \hat{r}) & d_{12}(\hat{p}) \\
d_{21}(\hat{p}, \hat{r} ; \alpha) & d_{22}(\hat{p}, \hat{r} ; \alpha)
\end{array}\right), \\
d_{11}(\hat{p}, \hat{r}) \stackrel{\text { def }}{=}-\hat{r}\left(\frac{\hat{p}}{K+\hat{p}}+\frac{K(1+\hat{p})}{(K+\hat{p})^{2}}+\Gamma_{M}-\Gamma_{R}\right)+\Gamma_{M}, \\
d_{12}(\hat{p}) \stackrel{\text { def }}{=}-\left(E_{M}+\lambda_{M} \Gamma_{M}-\lambda_{R} \Gamma_{R}+\frac{\hat{p}(1+\hat{p})}{K+\hat{p}}+\left(\Gamma_{M}-\Gamma_{R}\right) \hat{p}\right), \\
d_{21}(\hat{p}, \hat{r} ; \alpha) \stackrel{\text { def }}{=} \frac{K \hat{r}(\alpha-\hat{r})}{(K+\hat{p})^{2}}, \\
d_{22}(\hat{p}, \hat{r} ; \alpha) \stackrel{\text { def }}{=} \frac{\hat{p}(\alpha-2 \hat{r})}{K+\hat{p}}+\left(\Gamma_{M}-\Gamma_{R}\right)(1-2 \hat{r}) .
\end{gathered}
$$

The sum and product of its eigenvalues are equal to its trace and determinant, respectively. Hence, the optimal steady state $\left(\hat{p}_{\mathrm{opt}}^{*}, \hat{r}_{\mathrm{opt}}^{*}\right)$ is asymptotically stable under the following conditions.

Assumption 4.3

$\operatorname{tr} D\left(\hat{p}_{\mathrm{opt}}^{*}, \hat{r}_{\mathrm{opt}}^{*} ; \alpha_{\mathrm{opt}}^{*}\right)<0, \operatorname{det} D\left(\hat{p}_{\mathrm{opt}}^{*}, \hat{r}_{\mathrm{opt}}^{*} ; \alpha_{\mathrm{opt}}^{*}\right)>0$.

If $\Gamma_{M}=\Gamma_{R}=0$, this assumption allows a trivial analytical verification. For nonzero degradation rates, it can be checked numerically.

Let us summarize the obtained results in the form of a theorem.

\section{Theorem 4.1}

Let Assumptions 3.1-3.3, 4.1-4.3 hold. There exists a unique optimal steady state $\left(\hat{p}_{\text {opt }}^{*}, \hat{r}_{\text {opt }}^{*}\right) \in$ $G$ of (11) maximizing the growth rate (4). The first coordinate $\hat{p}_{\mathrm{opt}}^{*}$ is determined by (23),(24). The second coordinate $\hat{r}_{\mathrm{opt}}^{*}$ and corresponding control $\alpha_{\mathrm{opt}}^{*}$ are represented in terms of $\hat{p}_{\mathrm{opt}}^{*}$ according to (20),(21). The optimal steady state is a stable node or a stable focus.

We need one more assumption so as to have the function (20) strictly decreasing on $[0,+\infty$ ) (this property will be used when proving Theorem 5.1 in the next section).

\section{Assumption 4.4}

$\Gamma_{R}<\min \left(1, \frac{1}{K}\right)$.

\section{Proposition 4.1}

Under Assumptions 3.1-3.3, 4.1-4.4, the function (20) strictly decreases on $[0,+\infty)$. 
Proof

Direct calculations lead to the representation

$$
\begin{aligned}
& \frac{d \hat{r}^{*}\left(\hat{p}^{*}\right)}{d \hat{p}^{*}} \cdot\left(E_{M}+\lambda_{M} \Gamma_{M}-\lambda_{R} \Gamma_{R}\right.\left.+\frac{\hat{p}^{*}\left(1+\hat{p}^{*}\right)}{K+\hat{p}^{*}}+\left(\Gamma_{M}-\Gamma_{R}\right) \hat{p}^{*}\right)^{2} \\
&=-\frac{1}{\left(K+\hat{p}^{*}\right)^{2}} \cdot\left(\left(\left(1-\Gamma_{R}\right) E_{M}-\Gamma_{M}\left(1-K-\lambda_{M}+\left(\lambda_{M}-\lambda_{R}\right) \Gamma_{R}\right)\right) \cdot\left(\hat{p}^{*}\right)^{2}\right. \\
&+2 K\left(\left(1-\Gamma_{R}\right)\left(E_{M}+\lambda_{M} \Gamma_{M}\right)+\lambda_{R} \Gamma_{M} \Gamma_{R}\right) \cdot \hat{p}^{*} \\
&\left.+K\left(\left(1-\Gamma_{R} K\right)\left(E_{M}+\lambda_{M} \Gamma_{M}\right)+\lambda_{R} \Gamma_{M} \Gamma_{R} K\right)\right)
\end{aligned}
$$

for all $\hat{p}^{*} \geqslant 0$. Negativity of its right-hand side follows from Assumption 4.4 and the second inequality in Assumption 4.1.

\section{EXISTENCE OF OPTIMAL CONTROL STRATEGIES}

For proving existence of an optimal control strategy in the problem (11)-(14), additional conditions on the model parameters are required (also recall the comments after Assumption 4.1).

Assumption 5.1

The following inequalities hold:

$$
\begin{gathered}
\left(1-\alpha_{\mathrm{opt}}^{*}\right)\left(E_{M}+\lambda_{M} \Gamma_{M}-\lambda_{R} \Gamma_{R}\right)>\left(\Gamma_{M}-\Gamma_{R}\right)\left(1-\Gamma_{R} K\right), \\
\alpha_{\mathrm{opt}}^{*}>\Gamma_{R} .
\end{gathered}
$$

\section{Theorem 5.1}

Let Assumptions 3.1-3.3, 4.1-4.4, 5.1 hold. Then there exists an optimal control strategy in the problem (11)-(14) if the time horizon $\hat{T}$ is sufficiently large.

\section{Proof}

By virtue of the general existence result [39, Chapter VIII, $\S 1$, Theorem 1.1], it suffices to establish existence of a time horizon and an admissible control function such that the related state trajectory satisfies the terminal constraint (14).

Let $\bar{G}$ denote the closure of the strongly invariant domain $G$. In the set $\bar{G} \backslash\{(0,1)\}$, the system (11) with $\alpha \equiv \alpha_{\mathrm{opt}}^{*}$ has only one steady state $\left(\hat{p}_{\mathrm{opt}}^{*}, \hat{r}_{\mathrm{opt}}^{*}\right)$, and the latter is a stable node or a stable focus due to Theorem 4.1. If $\lambda_{R} \Gamma_{R}>0$, then $\left(\hat{p}_{\mathrm{opt}}^{*}, \hat{r}_{\mathrm{opt}}^{*}\right)$ is a unique steady state of this system in $\bar{G}$. If $\lambda_{R} \Gamma_{R}=0$, then there is also the steady state $(0,1)$, which is a saddle according to 
the representations

$$
\begin{gathered}
D\left(0,1 ; \alpha_{\mathrm{opt}}^{*}\right)=\left(\begin{array}{cc}
-\left(\frac{1}{K}-\Gamma_{R}\right) & -\left(E_{M}+\lambda_{M} \Gamma_{M}-\lambda_{R} \Gamma_{R}\right) \\
-\frac{1-\alpha_{\mathrm{opt}}^{*}}{K} & -\left(\Gamma_{M}-\Gamma_{R}\right)
\end{array}\right), \\
\operatorname{tr} D\left(0,1 ; \alpha_{\mathrm{opt}}^{*}\right)=-\left(\frac{1}{K}+\Gamma_{M}-2 \Gamma_{R}\right), \\
\operatorname{det} D\left(0,1 ; \alpha_{\mathrm{opt}}^{*}\right)=\left(\Gamma_{M}-\Gamma_{R}\right)\left(\frac{1}{K}-\Gamma_{R}\right)-\frac{1-\alpha_{\mathrm{opt}}^{*}}{K}\left(E_{M}+\lambda_{M} \Gamma_{M}-\lambda_{R} \Gamma_{R}\right)
\end{gathered}
$$

together with Assumptions 3.3, 4.4 and the first inequality in Assumption 5.1. For a saddle, the attracted set in its sufficiently small neighborhood consists only of the corresponding stable separatrix.

By using the general sufficient condition for local controllability [40, Chapter $6, \S 6.1$, Theorem 1], one can verify the following property for some open neighborhood $\Omega \subset G$ of the point $\left(\hat{p}_{\mathrm{opt}}^{*}, \hat{r}_{\mathrm{opt}}^{*}\right)$ : for any $\left(\hat{p}_{1}, \hat{r}_{1}\right) \in \Omega$, there exist a number $\hat{T}_{1}>0$ and a measurable control function $\alpha:\left[0, \hat{T}_{1}\right] \rightarrow$ $\left[\alpha_{\min }, \alpha_{\max }\right]$ such that the related state trajectory of (11) with the initial position $(\hat{p}(0), \hat{r}(0))=$ $\left(\hat{p}_{1}, \hat{r}_{1}\right)$ fulfills $\left(\hat{p}\left(\hat{T}_{1}\right), \hat{r}\left(\hat{T}_{1}\right)\right)=\left(\hat{p}_{\mathrm{opt}}^{*}, \hat{r}_{\mathrm{opt}}^{*}\right)$.

Now consider a state trajectory $\Phi$ of (11) with $\alpha \equiv \alpha_{\text {opt }}^{*}$ and $(\hat{p}(0), \hat{r}(0)) \in G$ on the infinite time interval $[0,+\infty)$, and suppose that $(0,1)$ is not its $\omega$-limit point. In compliance with the above reasonings, it suffices to prove that $\left(\hat{p}_{\mathrm{opt}}^{*}, \hat{r}_{\mathrm{opt}}^{*}\right)$ is an $\omega$-limit point of $\Phi$. Indeed, $(0,1)$ appears as a saddle in addition to the asymptotically stable steady state $\left(\hat{p}_{\mathrm{opt}}^{*}, \hat{r}_{\mathrm{opt}}^{*}\right)$ only when $\lambda_{R} \Gamma_{R}=0$. In this case, the stable separatrix of $(0,1)$ can be left (without any further entering) if $(\hat{p}, \hat{r}) \neq(0,1)$ and the control is changed to some constant value $\alpha \neq \alpha_{\mathrm{opt}}^{*}$ on a small time subinterval and then switched back to $\alpha=\alpha_{\mathrm{opt}}^{*}$. The subsequent arc with $\alpha=\alpha_{\mathrm{opt}}^{*}$ kept till infinity will not have $(0,1)$ as an $\omega$-limit point.

Note that, for every constant control $\alpha \in\left[\alpha_{\min }, \alpha_{\max }\right]$, the right-hand side of the system (11) is defined and continuously differentiable in some open neighborhood of the closed domain $\bar{G}$. Moreover, $\bar{\Phi} \subset \bar{G}$ due to Proposition 3.1. From Poincaré-Bendixson theorem [41, Chapter 3, §3.7, Theorem 1], Proposition 3.2, and the second inequality in Assumption 5.1, we conclude that $\left(\hat{p}_{\mathrm{opt}}^{*}, \hat{r}_{\mathrm{opt}}^{*}\right)$ will be an $\omega$-limit point of $\Phi$ if the system (11) with $\alpha \equiv \alpha_{\mathrm{opt}}^{*}$ does not have any periodic orbits in $\bar{G}$.

The state trajectory $\Phi$ lies outside some open neighborhood $\Omega_{(0,1)}$ of $(0,1)$, since this is not its $\omega$-limit point and $\Phi \subset G$. For the system (11), we have $d \hat{r} / d \hat{t}=0$ if $\hat{r}=0$, while, on the set

$$
\left\{(\hat{p}, \hat{r}) \in \partial G \backslash \Omega_{(0,1)}: \hat{r}>0\right\}
$$

the vector $(d \hat{p} / d \hat{t}, d \hat{r} / d \hat{t})$ is directed strictly inside $G$ if $\alpha<1$. Hence, if a periodic orbit of (11) with $\alpha \equiv \alpha_{\mathrm{opt}}^{*}$ exists in $\bar{G}$, then it should lie inside $G$. Thus, it remains to prove absence of such 
orbits in $G$. Let us introduce the function

$$
B(\hat{p}, \hat{r}) \stackrel{\text { def }}{=} \frac{1}{\hat{r}\left(E_{M}+\lambda_{M} \Gamma_{M}-\lambda_{R} \Gamma_{R}+\frac{\hat{p}(1+\hat{p})}{K+\hat{p}}+\left(\Gamma_{M}-\Gamma_{R}\right) \hat{p}\right)} \quad \forall(\hat{p}, \hat{r}) \in G
$$

and use Dulac's theorem [41, Chapter 3, §3.9, Theorem 2]. We obtain

$$
\begin{aligned}
& \frac{\partial}{\partial \hat{p}}\left(\left.B(\hat{p}, \hat{r}) \cdot \frac{d \hat{p}}{d \hat{t}}\right|_{(11)}\right)+\frac{\partial}{\partial \hat{r}}\left(\left.B(\hat{p}, \hat{r}) \cdot \frac{d \hat{r}}{d \hat{t}}\right|_{(11)}\right) \\
& \quad=\frac{1}{\hat{r}} \frac{d \hat{r}^{*}(\hat{p})}{d \hat{p}}-\frac{\frac{\hat{p}}{K+\hat{p}}+\Gamma_{M}-\Gamma_{R}}{E_{M}+\lambda_{M} \Gamma_{M}-\lambda_{R} \Gamma_{R}+\frac{\hat{p}(1+\hat{p})}{K+\hat{p}}+\left(\Gamma_{M}-\Gamma_{R}\right) \hat{p}}<0 \\
& \forall(\hat{p}, \hat{r}) \in G
\end{aligned}
$$

by virtue of Proposition 4.1 and Assumptions 3.3, 4.1. This completes the proof.

\section{Remark 5.1}

The minimum admissible $\hat{T}$ depends on the initial state (12). A heuristic way of choosing a suitable time horizon is to integrate the system (11) with $\alpha \equiv \alpha_{\text {opt }}^{*}$ numerically till a sufficiently small neighborhood of $\left(\hat{p}_{\mathrm{opt}}^{*}, \hat{r}_{\mathrm{opt}}^{*}\right)$ is reached and then to take $\hat{T}$ somewhat greater than the final instant of the integration.

\section{INVESTIGATION OF THE DYNAMIC OPTIMIZATION PROBLEM BY PONTRYAGIN'S MAXIMUM PRINCIPLE}

In optimal control theory, first-order necessary optimality conditions are given by Pontryagin's maximum principle or, in short, PMP [23]. For the problem (11)-(14) under the adopted assumptions, PMP leads to the Hamiltonian

$$
\begin{aligned}
& H\left(\hat{p}, \hat{r}, \alpha, \psi_{0}, \psi_{1}, \psi_{2}\right) \stackrel{\text { def }}{=} \psi_{1} \cdot\left((1-\hat{r}) E_{M}-\frac{\hat{p} \hat{r}}{K+\hat{p}}+\lambda_{M} \Gamma_{M}\right. \\
& \left.\quad-\left(\lambda_{M} \Gamma_{M}-\lambda_{R} \Gamma_{R}\right) \hat{r}-\hat{p}\left(\frac{\hat{p} \hat{r}}{K+\hat{p}}+\left(\Gamma_{M}-\Gamma_{R}\right) \hat{r}-\Gamma_{M}\right)\right) \\
& \quad+\psi_{2} \cdot\left(\alpha \frac{\hat{p} \hat{r}}{K+\hat{p}}-\Gamma_{R} \hat{r}-\hat{r}\left(\frac{\hat{p} \hat{r}}{K+\hat{p}}+\left(\Gamma_{M}-\Gamma_{R}\right) \hat{r}-\Gamma_{M}\right)\right) \\
& \quad-\psi_{0} \cdot\left(\frac{\hat{p} \hat{r}}{K+\hat{p}}+\left(\Gamma_{M}-\Gamma_{R}\right) \hat{r}-\Gamma_{M}\right) \\
& \forall\left(\hat{p}, \hat{r}, \alpha, \psi_{0}, \psi_{1}, \psi_{2}\right) \in G \times\left[\alpha_{\min }, \alpha_{\max }\right] \times \mathbb{R}^{3},
\end{aligned}
$$


adjoint system

$$
\begin{aligned}
& \left\{\begin{aligned}
\frac{\psi_{0} \equiv-1}{\frac{d \psi_{1}(\hat{t})}{d \hat{t}}} & \text { or } \psi_{0} \equiv 0, \\
& =\frac{\partial H\left(\hat{p}(\hat{t}), \hat{r}(\hat{t}), \alpha(\hat{t}), \psi_{0}, \psi_{1}(\hat{t}), \psi_{2}(\hat{t})\right)}{\partial \hat{p}} \\
& =\frac{K \hat{r}(\hat{t})}{(K+\hat{p}(\hat{t}))^{2}}\left(\psi_{1}(\hat{t})(1+\hat{p}(\hat{t}))+\psi_{2}(\hat{t})(\hat{r}(\hat{t})-\alpha(\hat{t}))+\psi_{0}\right)
\end{aligned}\right. \\
& +\psi_{1}(\hat{t})\left(\frac{\hat{p}(\hat{t}) \hat{r}(\hat{t})}{K+\hat{p}(\hat{t})}+\left(\Gamma_{M}-\Gamma_{R}\right) \hat{r}(\hat{t})-\Gamma_{M}\right), \\
& \frac{d \psi_{2}(\hat{t})}{d \hat{t}}=-\frac{\partial H\left(\hat{p}(\hat{t}), \hat{r}(\hat{t}), \alpha(\hat{t}), \psi_{0}, \psi_{1}(\hat{t}), \psi_{2}(\hat{t})\right)}{\partial \hat{r}} \\
& =\psi_{1}(\hat{t})\left(E_{M}+\lambda_{M} \Gamma_{M}-\lambda_{R} \Gamma_{R}\right) \\
& +\frac{\hat{p}(\hat{t})}{K+\hat{p}(\hat{t})}\left(\psi_{1}(\hat{t})(1+\hat{p}(\hat{t}))+\psi_{2}(\hat{t})(2 \hat{r}(\hat{t})-\alpha(\hat{t}))+\psi_{0}\right) \\
& +\left(\Gamma_{M}-\Gamma_{R}\right)\left(\psi_{1}(\hat{t}) \hat{p}(\hat{t})+\psi_{2}(\hat{t})(2 \hat{r}(\hat{t})-1)+\psi_{0}\right), \\
& \left(\psi_{0}, \psi_{1}(\hat{t}), \psi_{2}(\hat{t})\right) \neq(0,0,0) \quad \forall \hat{t} \in[0, \hat{T}],
\end{aligned}
$$

and Hamiltonian maximum condition

$$
\alpha(\hat{t})= \begin{cases}\alpha_{\min }, & \psi_{2}(\hat{t})<0, \\ \alpha_{\max }, & \psi_{2}(\hat{t})>0, \\ \text { arbitrary from }\left[\alpha_{\min }, \alpha_{\max }\right], & \psi_{2}(\hat{t})=0,\end{cases}
$$

which is necessarily fulfilled by an optimal control almost everywhere on $[0, \hat{T}]$ (with respect to Lebesgue measure). Absence of any terminal condition on the adjoint variables is called forth by the terminal condition (12) on the state variables.

An admissible process

$$
\mathcal{P}(\cdot)=\left(\hat{p}(\cdot), \hat{r}(\cdot), \alpha(\cdot), \psi_{0}, \psi_{1}(\cdot), \psi_{2}(\cdot)\right)
$$

satisfying PMP is called extremal. It is called normal if $\psi_{0} \neq 0$ and abnormal if $\psi_{0}=0$.

Since our controlled system is autonomous, the Hamiltonian (27) is conserved along any extremal process.

If the switching function $\psi_{2}(\cdot)$ vanishes over some time subinterval, then the corresponding control and part (arc) of the related extremal state trajectory are called singular [42].

The Hamiltonian maximum condition (30) directly implies the following result.

\section{Proposition 6.1}

Let Assumptions 3.1-3.3, 4.1-4.4, 5.1 hold, and let (31) be an extremal process for the problem (11)-(14). A switching of the control may appear only when $\psi_{2}(\hat{t})=0$. For such a 
switching instant, consider the expression

$$
\begin{aligned}
\left.\frac{d \psi_{2}(\hat{t})}{d \hat{t}}\right|_{\psi_{2}(\hat{t})=0} & =\psi_{1}(\hat{t}) \cdot\left(E_{M}+\lambda_{M} \Gamma_{M}-\lambda_{R} \Gamma_{R}+\frac{\hat{p}(\hat{t})(1+\hat{p}(\hat{t}))}{K+\hat{p}(\hat{t})}\right. \\
& \left.+\left(\Gamma_{M}-\Gamma_{R}\right) \hat{p}(\hat{t})\right)+\psi_{0} \cdot\left(\frac{\hat{p}(\hat{t})}{K+\hat{p}(\hat{t})}+\Gamma_{M}-\Gamma_{R}\right) .
\end{aligned}
$$

If (32) is negative, then the switching occurs in the direction from $\alpha=\alpha_{\max }$ to $\alpha=\alpha_{\min }$. If (32) is positive, then the opposite switching direction takes place.

\subsection{Characterization of singular arcs}

Consider a singular arc of an extremal process (31) in the problem (11)-(14). Along such an arc, the adjoint function $\psi_{2}(\cdot)$ and expression (32) vanish, which yields

$$
\psi_{1}(\hat{t})=-\psi_{0} \frac{\frac{\hat{p}(\hat{t})}{K+\hat{p}(\hat{t})}+\Gamma_{M}-\Gamma_{R}}{E_{M}+\lambda_{M} \Gamma_{M}-\lambda_{R} \Gamma_{R}+\frac{\hat{p}(\hat{t})(1+\hat{p}(\hat{t}))}{K+\hat{p}(\hat{t})}+\left(\Gamma_{M}-\Gamma_{R}\right) \hat{p}(\hat{t})},
$$

and the Hamiltonian (27) transforms into

$$
H\left(\hat{p}(\hat{t}), \hat{r}(\hat{t}), \alpha(\hat{t}), \psi_{0}, \psi_{1}(\hat{t}), \psi_{2}(\hat{t})\right)=\psi_{1}(\hat{t})\left(E_{M}+\lambda_{M} \Gamma_{M}+\Gamma_{M} \hat{p}(\hat{t})\right)+\psi_{0} \Gamma_{M} .
$$

If $\psi_{0}=0$, then, from $\psi_{2}(\hat{t})=0$ and (33), we obtain a contradiction with (29). This implies the next statement.

\section{Proposition 6.2}

Let Assumptions 3.1-3.3, 4.1-4.4, 5.1 hold. Abnormal extremal processes in the problem (11)-(14) cannot contain singular arcs.

Hence, the studied process $\mathcal{P}(\cdot)$ with a singular arc is normal, i. e., $\psi_{0}=-1$.

Due to the representation (34) and above-mentioned Hamiltonian conservation property, there exists a number $H_{\mathcal{P}(\cdot)}$ depending on the whole process $\mathcal{P}(\cdot)$ but not on time $\hat{t}$ and such that

$$
\psi_{1}(\hat{t})=\frac{H_{\mathcal{P}(\cdot)}+\Gamma_{M}}{E_{M}+\lambda_{M} \Gamma_{M}+\Gamma_{M} \hat{p}(\hat{t})}
$$

for the considered singular arc of $\mathcal{P}(\cdot)$. By setting the right-hand sides of (33) and (35) equal to each other, one can obtain a quadratic equation on $\hat{p}(\hat{t})$. Its coefficients are independent from $\hat{t}$, and it has at most two real solutions. This means that the singular arc stays at a steady state of (11), because $d \hat{p}(\hat{t}) / d \hat{t}=0$ leads to the representation (20) of $\hat{r}(\hat{t})$ in terms of $\hat{p}(\hat{t})$. A stronger result can indeed be established.

\section{Theorem 6.1}

Let Assumptions 3.1-3.3, 4.1-4.4, 5.1 hold. Singular arcs of extremal processes in the problem (11)-(14) stay at the optimal steady state $\left(\hat{p}_{\mathrm{opt}}^{*}, \hat{r}_{\mathrm{opt}}^{*}\right)$. 
Proof

Consider a subinterval $I \subseteq[0, \hat{T}]$ and a singular arc $(\hat{p}(\hat{t}), \hat{r}(\hat{t}))=\left(\hat{p}_{\text {sing }}^{*}, \hat{r}^{*}\left(\hat{p}_{\text {sing }}^{*}\right)\right), \hat{t} \in I$, of an extremal process (31) in the problem (11)-(14). The related adjoint functions satisfy

$$
\psi_{2}(\hat{t})=\frac{d \psi_{2}(\hat{t})}{d \hat{t}}=0, \quad \psi_{1}(\hat{t})=\frac{H_{\mathcal{P}(\cdot)}+\Gamma_{M}}{E_{M}+\lambda_{M} \Gamma_{M}+\Gamma_{M} \hat{p}_{\text {sing }}^{*}}, \quad \frac{d \psi_{1}(\hat{t})}{d \hat{t}}=0 \quad \forall \hat{t} \in I .
$$

Now take an arbitrary steady state $\left(\hat{p}^{*}, \hat{r}^{*}\left(\hat{p}^{*}\right)\right) \in G$ of (11) with the corresponding control $\alpha\left(\hat{p}^{*}\right)$ (recall the formulas $(20),(21))$; in particular, these can be $\left(\hat{p}_{\text {sing }}^{*}, \hat{r}^{*}\left(\hat{p}_{\text {sing }}^{*}\right)\right)$ and $\alpha^{*}\left(\hat{p}_{\text {sing }}^{*}\right)$, respectively. According to (22) and (27), we have

$$
H\left(\hat{p}^{*}, \hat{r}^{*}\left(\hat{p}^{*}\right), \alpha^{*}\left(\hat{p}^{*}\right),-1, \frac{H_{\mathcal{P}(\cdot)}+\Gamma_{M}}{E_{M}+\lambda_{M} \Gamma_{M}+\Gamma_{M} \hat{p}^{*}}, 0\right)=\hat{\mu}^{*}\left(\hat{p}^{*}\right),
$$

and, therefore,

$$
\frac{d}{d \hat{p}^{*}} H\left(\hat{p}^{*}, \hat{r}^{*}\left(\hat{p}^{*}\right), \alpha^{*}\left(\hat{p}^{*}\right),-1, \frac{H_{\mathcal{P}(\cdot)}+\Gamma_{M}}{E_{M}+\lambda_{M} \Gamma_{M}+\Gamma_{M} \hat{p}^{*}}, 0\right)=\frac{d \hat{\mu}^{*}\left(\hat{p}^{*}\right)}{d \hat{p}^{*}} .
$$

On the other hand, with the help of (36), we get

$$
\begin{aligned}
& \left.\frac{d}{d \hat{p}^{*}} H\left(\hat{p}^{*}, \hat{r}^{*}\left(\hat{p}^{*}\right), \alpha^{*}\left(\hat{p}^{*}\right),-1, \frac{H_{\mathcal{P}(\cdot)}+\Gamma_{M}}{E_{M}+\lambda_{M} \Gamma_{M}+\Gamma_{M} \hat{p}^{*}}, 0\right)\right|_{\hat{p}^{*}=\hat{p}_{\text {sing }}^{*}} \\
& =\frac{\partial H\left(\hat{p}_{\text {sing }}^{*}, \hat{r}^{*}\left(\hat{p}_{\text {sing }}^{*}\right), \alpha^{*}\left(\hat{p}_{\text {sing }}^{*}\right),-1, \frac{H_{\mathcal{P}(\cdot)}+\Gamma_{M}}{E_{M}+\lambda_{M} \Gamma_{M}+\Gamma_{M} \hat{p}_{\text {sing }}^{*}}, 0\right)}{\partial \hat{p}} \\
& +\frac{\partial H\left(\hat{p}_{\text {sing }}^{*}, \hat{r}^{*}\left(\hat{p}_{\text {sing }}^{*}\right), \alpha^{*}\left(\hat{p}_{\text {sing }}^{*}\right),-1, \frac{H_{\mathcal{P}(\cdot)}+\Gamma_{M}}{E_{M}+\lambda_{M} \Gamma_{M}+\Gamma_{M} \hat{p}_{\text {sing }}^{*}}, 0\right)}{\partial \hat{r}} \cdot \frac{d \hat{r}^{*}\left(\hat{p}_{\text {sing }}^{*}\right)}{d \hat{p}^{*}} \\
& +\frac{\partial H\left(\hat{p}_{\text {sing }}^{*}, \hat{r}^{*}\left(\hat{p}_{\text {sing }}^{*}\right), \alpha^{*}\left(\hat{p}_{\text {sing }}^{*}\right),-1, \frac{H_{\mathcal{P}(\cdot)+\Gamma_{M}}}{E_{M}+\lambda_{M} \Gamma_{M}+\Gamma_{M} \hat{p}_{\text {sing }}^{*}}, 0\right)}{\partial \alpha} \cdot \frac{d \alpha^{*}\left(\hat{p}_{\text {sing }}^{*}\right)}{d \hat{p}^{*}} \\
& +\frac{\partial H\left(\hat{p}_{\text {sing }}^{*}, \hat{r}^{*}\left(\hat{p}_{\text {sing }}^{*}\right), \alpha^{*}\left(\hat{p}_{\text {sing }}^{*}\right),-1, \frac{H_{\mathcal{P}(\cdot)}+\Gamma_{M}}{E_{M}+\lambda_{M} \Gamma_{M}+\Gamma_{M} \hat{p}_{\text {sing }}^{*}}, 0\right)}{\partial \psi_{1}} . \\
& \cdot\left(\left.\frac{d}{d \hat{p}^{*}}\left(\frac{H_{\mathcal{P}(\cdot)}+\Gamma_{M}}{E_{M}+\lambda_{M} \Gamma_{M}+\Gamma_{M} \hat{p}^{*}}\right)\right|_{\hat{p}^{*}=\hat{p}_{\text {sing }}^{*}}\right) \\
& =-\frac{d \psi_{1}(\hat{t})}{d \hat{t}}-\frac{d \psi_{2}(\hat{t})}{d \hat{t}} \cdot \frac{d \hat{r}^{*}\left(\hat{p}_{\text {sing }}^{*}\right)}{d \hat{p}^{*}}+\psi_{2}(\hat{t}) \cdot \frac{\hat{p}_{\text {sing }}^{*} \hat{r}^{*}\left(\hat{p}_{\text {sing }}^{*}\right)}{K+\hat{p}_{\text {sing }}^{*}} \cdot \frac{d \alpha^{*}\left(\hat{p}_{\text {sing }}^{*}\right)}{d \hat{p}^{*}} \\
& +0 \cdot\left(\left.\frac{d}{d \hat{p}^{*}}\left(\frac{H_{\mathcal{P}(\cdot)}+\Gamma_{M}}{E_{M}+\lambda_{M} \Gamma_{M}+\Gamma_{M} \hat{p}^{*}}\right)\right|_{\hat{p}^{*}=\hat{p}_{\text {sing }}^{*}}\right)=0
\end{aligned}
$$

$\forall \hat{t} \in I$.

Thus, $d \hat{\mu}^{*}\left(\hat{p}_{\text {sing }}^{*}\right) / d \hat{p}^{*}=0$, and, due to $(25)$, we conclude that $\hat{p}_{\text {sing }}^{*}=\hat{p}_{\text {opt }}^{*}$ and $\hat{r}^{*}\left(\hat{p}_{\text {sing }}^{*}\right)=$ $\hat{r}^{*}\left(\hat{p}_{\mathrm{opt}}^{*}\right)=\hat{r}_{\mathrm{opt}}^{*}$.

One can directly verify that the switching function $\psi_{2}=\psi_{2}(\hat{t})$ should be consecutively differentiated four times before an explicit appearance of the control $\alpha=\alpha(\hat{t})$ in the corresponding 
expressions obtained by using the equations (11),(28) with the substituted singular arc condition $\psi_{2}(\hat{t})=0$. The Kelley condition [42] or, in other words, the generalized Legendre-Clebsch condition [25] (which is necessary for optimality of singular arcs) also holds there. In compliance with the theory of chattering control $[24,25,43]$, we arrive at the following property.

\section{Theorem 6.2}

Let Assumptions 3.1-3.3, 4.1-4.4, 5.1 hold. Singular arcs of extremal processes in the problem (11)-(14) have the second order and can be entered or left only by chattering (i.e., with infinite numbers of bang-bang switchings between the boundary control values $\alpha_{\min }, \alpha_{\max }$ on a finite time interval).

Note that singular arcs play a central role in dynamic optimization problems arising in many applied areas such as economics, engineering, ecology, biology, and medical sciences [24,25,32,4446]. Similarly to $[8,17]$, let us exclude abnormal extremal processes from the further consideration of the problem (11)-(14) according to the fact that they do not allow singular arcs (Proposition 6.2) and, therefore, do not admit a clear biological interpretation.

\subsection{Characterization of chattering arcs}

In the problem (11)-(14), a chattering phenomenon can be described by the following local phase portrait on the plane $(\hat{p}, \hat{r})$ of the state variables (see Fig. 2 , the general theory is given in $[24,25,43]$ ):

1) $N$ is some open neighborhood of the point $\left(\hat{p}_{\mathrm{opt}}^{*}, \hat{r}_{\mathrm{opt}}^{*}\right)$;

2) a continuous curve $\Sigma$ divides $N$ into two open half-neighborhoods $N_{0}$ and $N_{1}, N=N_{0} \cup$ $\Sigma \cup N_{1}$;

3) the point $\left(\hat{p}_{\mathrm{opt}}^{*}, \hat{r}_{\mathrm{opt}}^{*}\right)$ lies on $\Sigma$ and divides $\Sigma$ into two regular parameterized curves $\Sigma_{0}$ and $\Sigma_{1}, \Sigma=\Sigma_{0} \cup\left\{\left(\hat{p}_{\text {opt }}^{*}, \hat{r}_{\text {opt }}^{*}\right)\right\} \cup \Sigma_{1}$;

4) the curves $\Sigma_{0} \cup\left\{\left(\hat{p}_{\text {opt }}^{*}, \hat{r}_{\text {opt }}^{*}\right)\right\}$ and $\Sigma_{1} \cup\left\{\left(\hat{p}_{\text {opt }}^{*}, \hat{r}_{\text {opt }}^{*}\right)\right\}$ with the included endpoint $\left(\hat{p}_{\mathrm{opt}}^{*}, \hat{r}_{\mathrm{opt}}^{*}\right)$ also allow regular parametrizations and, consequently, have tangent lines at this endpoint;

5) $\Sigma_{0}$ and $\Sigma_{1}$ are constituted by switching points of extremal state trajectories in the directions from $\alpha=\alpha_{\max }$ to $\alpha=\alpha_{\min }$ and from $\alpha=\alpha_{\min }$ to $\alpha=\alpha_{\max }$, respectively;

6) the vector of the right-hand side of the system (11) is directed strictly inside $N_{0}$ (i. e., strictly outside $N_{1}$ ) on $\Sigma_{0}$ and strictly inside $N_{1}$ (i.e., strictly outside $N_{0}$ ) on $\Sigma_{1}$ for $\alpha \in\left\{\alpha_{\min }, \alpha_{\max }\right\}$ and, therefore, for all $\alpha \in\left[\alpha_{\min }, \alpha_{\max }\right]$;

7) extremal state trajectories cross the curves $\Sigma_{0}$ and $\Sigma_{1}$ infinitely many times before entering the singular arc at $\left(\hat{p}_{\mathrm{opt}}^{*}, \hat{r}_{\mathrm{opt}}^{*}\right)$, and all of these crossings are transversal (nontangential) due to the previous item;

8) the corresponding local feedback map can be determined as $\alpha=\alpha_{\min }$ in $N_{0}$ and $\alpha=\alpha_{\max }$ in $N_{1}$. 


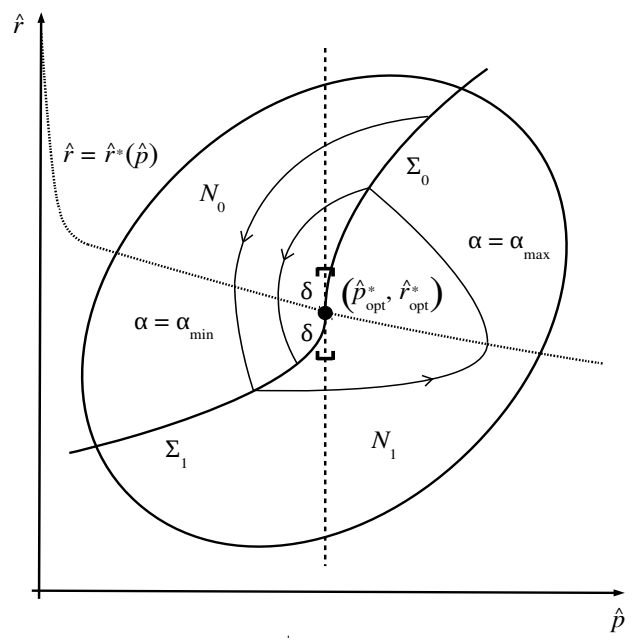

Figure 2. Chattering phenomenon on the plane $(\hat{p}, \hat{r})$ in the problem (11)-(14). The directions of arrows on the state trajectories correspond to motion in forward time $\hat{t}$. There is a unique tangent line to $\Sigma$ at $\left(\hat{p}_{\mathrm{opt}}^{*}, \hat{r}_{\mathrm{opt}}^{*}\right)$, and it is vertical.

Note that $\alpha(\cdot)$ does not explicitly appear in the first equation of (11). Then the first component of the velocity vector field is continuous regardless of a chosen control law. Since $d \hat{p} /\left.d \hat{t}\right|_{(11),(\hat{p}, \hat{r})=\left(\hat{p}_{\mathrm{opt}}^{*}, \hat{r}_{\mathrm{opt}}^{*}\right)}=0$, we come to the intuitive hypothesis that the tangent line to the switching curve $\Sigma$ at the point $\left(\hat{p}_{\text {opt }}^{*}, \hat{r}_{\text {opt }}^{*}\right)$ should be unique and vertical (as depicted in Fig. 2). This can indeed be verified by the following property related to the framework of generalized characteristics for first-order partial differential equations [43,47]. If the directions specified by the vectors

$$
\begin{aligned}
\left.\left(\frac{\partial H}{\partial \psi_{1}}, \frac{\partial H}{\partial \psi_{2}}\right)\right|_{(27),(\hat{p}, \hat{r})=\left(\hat{p}_{\mathrm{opt}}^{*}, \hat{r}_{\mathrm{opt}}^{*}\right), \alpha=\alpha_{\min }} & =\left.\left(\frac{d \hat{p}}{d \hat{t}}, \frac{d \hat{r}}{d \hat{t}}\right)\right|_{(11),(\hat{p}, \hat{r})=\left(\hat{p}_{\mathrm{opt}}^{*}, \hat{r}_{\mathrm{opt}}^{*}\right), \alpha=\alpha_{\mathrm{min}}}, \\
\left.\left(\frac{\partial H}{\partial \psi_{1}}, \frac{\partial H}{\partial \psi_{2}}\right)\right|_{(27),(\hat{p}, \hat{r})=\left(\hat{p}_{\mathrm{opt}}^{*}, \hat{r}_{\mathrm{opt}}^{*}\right), \alpha=\alpha_{\max }} & =\left.\left(\frac{d \hat{p}}{d \hat{t}}, \frac{d \hat{r}}{d \hat{t}}\right)\right|_{(11),(\hat{p}, \hat{r})=\left(\hat{p}_{\mathrm{opt}}^{*}, \hat{r}_{\mathrm{opt}}^{*}\right), \alpha=\alpha_{\mathrm{max}}}
\end{aligned}
$$

coincide with each other, then this is in fact the direction of the tangent line to $\Sigma$ at $\left(\hat{p}_{\mathrm{opt}}^{*}, \hat{r}_{\mathrm{opt}}^{*}\right)$. The latter is vertical, because, as mentioned above, we have

$$
\left.\frac{d \hat{p}}{d \hat{t}}\right|_{(11),(\hat{p}, \hat{r})=\left(\hat{p}_{\mathrm{opt}}^{*}, \hat{r}_{\mathrm{opt}}^{*}\right), \alpha=\alpha_{\min }}=\left.\frac{d \hat{p}}{d \hat{t}}\right|_{(11),(\hat{p}, \hat{r})=\left(\hat{p}_{\mathrm{opt}}^{*}, \hat{r}_{\mathrm{opt}}^{*}\right), \alpha=\alpha_{\max }}=0 .
$$

Note that, for well-known Fuller and Marshal problems, such tangent lines are also vertical [24,25].

Furthermore, for all chattering arcs which enter the singular arc at $\left(\hat{p}_{\mathrm{opt}}^{*}, \hat{r}_{\mathrm{opt}}^{*}\right)$, the conserved Hamiltonian value is the same and equal to

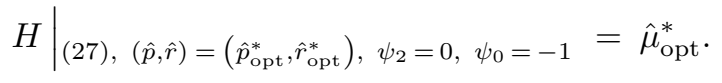


Let (31) be a normal extremal process containing such a chattering arc, and let $\hat{t}=\hat{t}_{\mathrm{sw}}$ be one of the corresponding switching instants, i.e.,

$$
\begin{gathered}
\psi_{2}\left(\hat{t}_{\mathrm{sw}}\right)=0 \\
H\left(\hat{p}\left(\hat{t}_{\mathrm{sw}}\right), \hat{r}\left(\hat{t}_{\mathrm{sw}}\right), \alpha\left(\hat{t}_{\mathrm{sw}}\right),-1, \psi_{1}\left(\hat{t}_{\mathrm{sw}}\right), 0\right)=\hat{\mu}_{\mathrm{opt}}^{*} .
\end{gathered}
$$

If $d \hat{p}\left(\hat{t}_{\mathrm{sw}}\right) / d \hat{t}=0$, then $\hat{r}\left(\hat{t}_{\mathrm{sw}}\right)=\hat{r}^{*}\left(\hat{p}\left(\hat{t}_{\mathrm{sw}}\right)\right)$ and, from (37), we get $\hat{\mu}^{*}\left(\hat{p}\left(\hat{t}_{\mathrm{sw}}\right)\right)=\hat{\mu}_{\mathrm{opt}}^{*}$, which yields $\left(\hat{p}\left(\hat{t}_{\mathrm{sw}}\right), \hat{r}\left(\hat{t}_{\mathrm{sw}}\right)\right)=\left(\hat{p}_{\mathrm{opt}}^{*}, \hat{r}_{\mathrm{opt}}^{*}\right)$, i. e., at $\hat{t}=\hat{t}_{\mathrm{sw}}$, this is already a singular arc. Hence, the case $d \hat{p}\left(\hat{t}_{\mathrm{sw}}\right) / d \hat{t}=0$ can be taken out of the chattering arcs' consideration, and we suppose that $d \hat{p}\left(\hat{t}_{\mathrm{sw}}\right) / d \hat{t} \neq 0$. Then (37) implies

$$
\begin{aligned}
\psi_{1}\left(\hat{t}_{\mathrm{sw}}\right)= & \left(1-\hat{r}\left(\hat{t}_{\mathrm{sw}}\right)\right) E_{M}-\frac{\hat{p}\left(\hat{t}_{\mathrm{sw}}\right) \hat{r}\left(\hat{t}_{\mathrm{sw}}\right)}{K+\hat{p}\left(\hat{t}_{\mathrm{sw}}\right)} \\
& +\lambda_{M} \Gamma_{M}-\left(\lambda_{M} \Gamma_{M}-\lambda_{R} \Gamma_{R}\right) \hat{r}\left(\hat{t}_{\mathrm{sw}}\right) \\
& \left.\quad-\hat{p}\left(\hat{t}_{\mathrm{sw}}\right)\left(\frac{\hat{p}\left(\hat{t}_{\mathrm{sw}}\right) \hat{r}\left(\hat{t}_{\mathrm{sw}}\right)}{K+\hat{p}\left(\hat{t}_{\mathrm{sw}}\right)}+\left(\Gamma_{M}-\Gamma_{R}\right) \hat{r}\left(\hat{t}_{\mathrm{sw}}\right)-\Gamma_{M}\right)\right)^{-1} . \\
& \cdot\left(\hat{\mu}_{\mathrm{opt}}^{*}+\Gamma_{M}-\left(\frac{\hat{p}\left(\hat{t}_{\mathrm{sw}}\right)}{K+\hat{p}\left(\hat{t}_{\mathrm{sw}}\right)}+\Gamma_{M}-\Gamma_{R}\right) \hat{r}\left(\hat{t}_{\mathrm{sw}}\right)\right) .
\end{aligned}
$$

For the chattering arcs, Proposition 6.1 can be strengthened by the following result (see Fig. 2).

\section{Theorem 6.3}

Let Assumptions 3.1-3.3, 4.1-4.4, 5.1 hold. For a chattering arc of a normal extremal process (31) in the problem (11)-(14), switchings in the direction from $\alpha=\alpha_{\max }$ to $\alpha=\alpha_{\min }$ may appear only in the domain

$$
\left\{(\hat{p}, \hat{r}) \in G: \hat{r}>\hat{r}^{*}(\hat{p})\right\},
$$

and switchings in the opposite direction may happen only in the domain

$$
\left\{(\hat{p}, \hat{r}) \in G: \hat{r}<\hat{r}^{*}(\hat{p})\right\} .
$$


Proof

After substituting (38) into (32), we get

$$
\begin{aligned}
& \frac{d \psi_{2}\left(\hat{t}_{\mathrm{sw}}\right)}{d \hat{t}}=\left(\left(1-\hat{r}\left(\hat{t}_{\mathrm{sw}}\right)\right) E_{M}-\frac{\hat{p}\left(\hat{t}_{\mathrm{sw}}\right) \hat{r}\left(\hat{t}_{\mathrm{sw}}\right)}{K+\hat{p}\left(\hat{t}_{\mathrm{sw}}\right)}\right. \\
& +\lambda_{M} \Gamma_{M}-\left(\lambda_{M} \Gamma_{M}-\lambda_{R} \Gamma_{R}\right) \hat{r}\left(\hat{t}_{\mathrm{sw}}\right) \\
& \left.-\hat{p}\left(\hat{t}_{\mathrm{sw}}\right)\left(\frac{\hat{p}\left(\hat{t}_{\mathrm{sw}}\right) \hat{r}\left(\hat{t}_{\mathrm{sw}}\right)}{K+\hat{p}\left(\hat{t}_{\mathrm{sw}}\right)}+\left(\Gamma_{M}-\Gamma_{R}\right) \hat{r}\left(\hat{t}_{\mathrm{sw}}\right)-\Gamma_{M}\right)\right)^{-1} . \\
& \cdot\left(( \hat { \mu } _ { \mathrm { opt } } ^ { * } + \Gamma _ { M } ) \cdot \left(E_{M}+\lambda_{M} \Gamma_{M}-\lambda_{R} \Gamma_{R}+\frac{\hat{p}\left(\hat{t}_{\mathrm{sw}}\right)\left(1+\hat{p}\left(\hat{t}_{\mathrm{sw}}\right)\right)}{K+\hat{p}\left(\hat{t}_{\mathrm{sw}}\right)}\right.\right. \\
& \left.+\left(\Gamma_{M}-\Gamma_{R}\right) \hat{p}\left(\hat{t}_{\mathrm{sw}}\right)\right)-\left(\frac{\hat{p}\left(\hat{t}_{\mathrm{sw}}\right)}{K+\hat{p}\left(\hat{t}_{\mathrm{sw}}\right)}+\Gamma_{M}-\Gamma_{R}\right) . \\
& \left.\cdot\left(E_{M}+\lambda_{M} \Gamma_{M}+\Gamma_{M} \hat{p}\left(\hat{t}_{\mathrm{sw}}\right)\right)\right) \text {. }
\end{aligned}
$$

With the help of the representations (20),(22), the last formula can be transformed into

$$
\begin{aligned}
\frac{d \psi_{2}\left(\hat{t}_{\mathrm{sw}}\right)}{d \hat{t}}= & \left(E_{M}+\lambda_{M} \Gamma_{M}-\lambda_{R} \Gamma_{R}+\frac{\hat{p}\left(\hat{t}_{\mathrm{sw}}\right)\left(1+\hat{p}\left(\hat{t}_{\mathrm{sw}}\right)\right)}{K+\hat{p}\left(\hat{t}_{\mathrm{sw}}\right)}\right. \\
& \left.+\left(\Gamma_{M}-\Gamma_{R}\right) \hat{p}\left(\hat{t}_{\mathrm{sw}}\right)\right)^{-1} \cdot\left(\hat{r}^{*}\left(\hat{p}\left(\hat{t}_{\mathrm{sw}}\right)\right)-\hat{r}\left(\hat{t}_{\mathrm{sw}}\right)\right)^{-1} \cdot \\
& \quad\left(\hat{\mu}_{\mathrm{opt}}^{*}+\Gamma_{M}\right) \cdot\left(E_{M}+\lambda_{M} \Gamma_{M}-\lambda_{R} \Gamma_{R}+\frac{\hat{p}\left(\hat{t}_{\mathrm{sw}}\right)\left(1+\hat{p}\left(\hat{t}_{\mathrm{sw}}\right)\right)}{K+\hat{p}\left(\hat{t}_{\mathrm{sw}}\right)}\right. \\
& \left.\quad+\left(\Gamma_{M}-\Gamma_{R}\right) \hat{p}\left(\hat{t}_{\mathrm{sw}}\right)\right)-\left(\frac{\hat{p}\left(\hat{t}_{\mathrm{sw}}\right)}{K+\hat{p}\left(\hat{t}_{\mathrm{sw}}\right)}+\Gamma_{M}-\Gamma_{R}\right) . \\
& \left.\quad \cdot\left(E_{M}+\lambda_{M} \Gamma_{M}+\Gamma_{M} \hat{p}\left(\hat{t}_{\mathrm{sw}}\right)\right)\right) \\
= & \left(\hat{r}^{*}\left(\hat{p}\left(\hat{t}_{\mathrm{sw}}\right)\right)-\hat{r}\left(\hat{t}_{\mathrm{sw}}\right)\right)^{-1} \cdot\left(\hat{\mu}_{\mathrm{opt}}^{*}-\hat{\mu}^{*}\left(\hat{p}\left(\hat{t}_{\mathrm{sw}}\right)\right)\right) .
\end{aligned}
$$

Recall that $\hat{p}_{\text {opt }}^{*}$ is a unique maximum point of the function $\hat{\mu}^{*}=\hat{\mu}^{*}\left(\hat{p}^{*}\right)$ on the interval $[0,+\infty)$. Now, from Proposition 6.1, we conclude that, for completing the proof, it remains to analyze the case $\hat{p}\left(\hat{t}_{\mathrm{sw}}\right)=\hat{p}_{\mathrm{opt}}^{*}$ under the conditions $\psi_{2}\left(\hat{t}_{\mathrm{sw}}\right)=d \psi_{2}\left(\hat{t}_{\mathrm{sw}}\right) / d \hat{t}=0$.

For any constant control $\alpha$ (such as $\alpha=\alpha_{\min }$ or $\alpha=\alpha_{\max }$ ), one can represent $d^{2} \psi_{2}(\hat{t}) / d \hat{t}^{2}$ in terms of $\hat{p}(\hat{t}), \hat{r}(\hat{t}), \alpha, \psi_{1}(\hat{t}), \psi_{2}(\hat{t})$ subject to the state dynamic and adjoint equations (11),(28). In the corresponding expression, let us set $\hat{t}=\hat{t}_{\mathrm{sw}}, \psi_{2}\left(\hat{t}_{\mathrm{sw}}\right)=0$ and substitute the formula (33) (following from $\psi_{2}\left(\hat{t}_{\mathrm{sw}}\right)=d \psi_{2}\left(\hat{t}_{\mathrm{sw}}\right) / d \hat{t}=0$ ). Then the control will disappear there. Moreover, let us take $\hat{p}\left(\hat{t}_{\mathrm{sw}}\right)=\hat{p}_{\mathrm{opt}}^{*}$. Finally, we will obtain a linear expression with respect to $\hat{r}\left(\hat{t}_{\mathrm{sw}}\right)$, which is zero for $\hat{r}\left(\hat{t}_{\mathrm{sw}}\right)=\hat{r}^{*}\left(\hat{p}_{\mathrm{opt}}^{*}\right)=\hat{r}_{\mathrm{opt}}^{*}$ (due to the characterization of singular arcs in Subsection 6.1), negative for $\hat{r}\left(\hat{t}_{\mathrm{sw}}\right)>\hat{r}_{\mathrm{opt}}^{*}$, and positive for $\hat{r}\left(\hat{t}_{\mathrm{sw}}\right)<\hat{r}_{\mathrm{opt}}^{*}$. This yields the desired result, since $\left(\hat{p}\left(\hat{t}_{\mathrm{sw}}\right), \hat{r}\left(\hat{t}_{\mathrm{sw}}\right)\right) \neq\left(\hat{p}_{\mathrm{opt}}^{*}, \hat{r}_{\mathrm{opt}}^{*}\right)$. 


\section{Remark 6.1}

The proof of Theorem 6.3 relies on PMP and does not use specific conditions on $N_{0}, N_{1}, \Sigma, \Sigma_{0}, \Sigma_{1}$ formulated in the beginning of this subsection.

The next section develops a numerical technique to construct the chattering switching curve in the studied problem.

\section{NUMERICAL METHOD TO APPROXIMATE THE CHATTERING SWITCHING CURVE}

Based on the reasonings of the previous section, the following numerical method to approximate the chattering switching curve $\Sigma$ in the problem (11)-(14) can be proposed.

Fix some numbers $\hat{p}_{\text {min }} \geqslant 0, \hat{p}_{\max }>\hat{p}_{\min }, \hat{r}_{\min } \in[0,1), \hat{r}_{\max } \in\left(\hat{r}_{\min }, 1\right]$ determining the bounded domain

$$
\left\{(\hat{p}, \hat{r}): \hat{p}_{\min }<\hat{p}<\hat{p}_{\max }, \hat{r}_{\min }<\hat{r}<\hat{r}_{\max }\right\}
$$

in which $\Sigma$ is going to be approximated. The optimal steady state $\left(\hat{p}_{\mathrm{opt}}^{*}, \hat{r}_{\mathrm{opt}}^{*}\right)$ should lie there.

Take a sufficiently small number $\delta>0$ and consider the vertical line segment

$$
L_{\delta} \stackrel{\text { def }}{=}\left\{\left(\hat{p}_{\mathrm{opt}}^{*}, \hat{r}\right): \hat{r}_{\mathrm{opt}}^{*}-\delta \leqslant \hat{r} \leqslant \hat{r}_{\mathrm{opt}}^{*}+\delta\right\}
$$

as an approximation of a small part of $\Sigma$ near the point $\left(\hat{p}_{\mathrm{opt}}^{*}, \hat{r}_{\mathrm{opt}}^{*}\right)$ (see Fig. 2 and recall that the tangent line to $\Sigma$ at $\left(\hat{p}_{\mathrm{opt}}^{*}, \hat{r}_{\mathrm{opt}}^{*}\right)$ is vertical). The upper half of $L_{\delta}$ with the excluded center $\left(\hat{p}_{\mathrm{opt}}^{*}, \hat{r}_{\mathrm{opt}}^{*}\right)$ lies in the region (39), and the lower one is contained in (40). Discretize $L_{\delta} \backslash\left\{\left(\hat{p}_{\mathrm{opt}}^{*}, \hat{r}_{\mathrm{opt}}^{*}\right)\right\}$ by the grid consisting of the points

$$
Q_{i} \stackrel{\text { def }}{=}\left(\hat{p}_{\mathrm{opt}}^{*}, \hat{r}_{\mathrm{opt}}^{*}+i \frac{\delta}{n}\right), \quad i \in \mathcal{I} \stackrel{\text { def }}{=}\{-n,-n+1, \ldots, n-1, n\} \backslash\{0\},
$$

for a sufficiently large $n \in \mathbb{N}$. Rewrite the system of the state dynamic and adjoint equations (11),(28) in reverse time $\hat{\tau} \stackrel{\text { def }}{=} \hat{T}-\hat{t}$ with

$$
\alpha= \begin{cases}\alpha_{\min }, & \psi_{2}<0, \\ \alpha_{\max }, & \psi_{2}>0, \\ \alpha_{\min }, & \psi_{2}=0,\left.\quad \frac{d \psi_{2}}{d \hat{t}}\right|_{\psi_{2}=0}<0, \\ \alpha_{\max }, & \psi_{2}=0,\left.\frac{d \psi_{2}}{d \hat{t}}\right|_{\psi_{2}=0}>0, \\ \alpha_{\min }, & \psi_{2}=\left.\frac{d \psi_{2}}{d \hat{t}}\right|_{\psi_{2}=0}=0, \hat{r}>\hat{r}^{*}(\hat{p}), \\ \alpha_{\max }, & \psi_{2}=\left.\frac{d \psi_{2}}{d \hat{t}}\right|_{\psi_{2}=0}=0, \hat{r}<\hat{r}^{*}(\hat{p}) .\end{cases}
$$

The expression (32) with $\psi_{0}=-1$ should be substituted in (42). The case $\psi_{2}=d \psi_{2} /\left.d \hat{t}\right|_{\psi_{2}=0}=0$, $\hat{r}=\hat{r}^{*}(\hat{p})$ is excluded there, because it refers to the state $\left(\hat{p}_{\text {opt }}^{*}, \hat{r}_{\text {opt }}^{*}\right)$ (recall the considerations coming before Theorem 6.3). Since the constructed system is autonomous, the initial time instant 
for its integration can be chosen as $\hat{\tau}=0$. For $i \in \mathcal{I}$, specify the initial conditions at $\hat{\tau}=0$ as

$$
\begin{aligned}
\left(\hat{p}_{i}^{0}, \hat{r}_{i}^{0}\right) \stackrel{\text { def }}{=} & Q_{i}, \quad \psi_{2, i}^{0} \stackrel{\text { def }}{=} 0 \\
\psi_{1, i}^{0} \stackrel{\text { def }}{=} & \left(\left(1-\hat{r}_{i}^{0}\right) E_{M}-\frac{\hat{p}_{i}^{0} \hat{r}_{i}^{0}}{K+\hat{p}_{i}^{0}}+\lambda_{M} \Gamma_{M}-\left(\lambda_{M} \Gamma_{M}-\lambda_{R} \Gamma_{R}\right) \hat{r}_{i}^{0}\right. \\
& \left.\quad-\hat{p}_{i}^{0}\left(\frac{\hat{p}_{i}^{0} \hat{r}_{i}^{0}}{K+\hat{p}_{i}^{0}}+\left(\Gamma_{M}-\Gamma_{R}\right) \hat{r}_{i}^{0}-\Gamma_{M}\right)\right)^{-1} \cdot \\
& \cdot\left(\hat{\mu}_{\mathrm{opt}}^{*}+\Gamma_{M}-\left(\frac{\hat{p}_{i}^{0}}{K+\hat{p}_{i}^{0}}+\Gamma_{M}-\Gamma_{R}\right) \hat{r}_{i}^{0}\right)
\end{aligned}
$$

(the last equality follows from (38)). Let

$$
\left(\hat{p}_{i}(\hat{\tau}), \hat{r}_{i}(\hat{\tau}), \psi_{1, i}(\hat{\tau}), \psi_{2, i}(\hat{\tau})\right), \quad \hat{\tau} \in\left[0, \hat{T}_{i}\right), \quad i \in \mathcal{I}
$$

be the family of the corresponding solutions to the introduced system, so that, for any $i \in \mathcal{I}$, the time horizon $\hat{T}_{i}>0$ equals either the instant of the first exit from the domain (41) or $+\infty$ if there is no such an exit. Then the set

$$
\bigcup_{i \in \mathcal{I}}\left\{\left(\hat{p}_{i}(\hat{\tau}), \hat{r}_{i}(\hat{\tau})\right): \hat{\tau} \in\left[0, \hat{T}_{i}\right), \psi_{2, i}(\hat{\tau})=0\right\}
$$

can serve as an approximation of $\Sigma$. From the computational point of view, here it is reasonable to replace $\hat{T}_{i}$ with $\min \left(\hat{T}_{i}, \Theta\right)$, where $\Theta \in(0,+\infty)$ is a sufficiently large finite number.

\section{Remark 7.1}

In the local phase portrait described in the beginning of Subsection 6.2, chattering arcs are unique solutions to the differential inclusion with the multi-valued right-hand side specified by the righthand side of (11) and by the condition

$$
\alpha(\hat{t}) \in \begin{cases}\left\{\alpha_{\min }\right\}, & (\hat{p}(\hat{t}), \hat{r}(\hat{t})) \in N_{0}, \\ \left\{\alpha_{\max }\right\}, & (\hat{p}(\hat{t}), \hat{r}(\hat{t})) \in N_{1}, \\ {\left[\alpha_{\min }, \alpha_{\max }\right],} & (\hat{p}(\hat{t}), \hat{r}(\hat{t})) \in \Sigma .\end{cases}
$$

Indeed, due to the item 6 of that description, the control values on $\Sigma_{0} \cup \Sigma_{1}$ can be chosen arbitrarily from $\left[\alpha_{\min }, \alpha_{\max }\right]$. Besides, the control values at the point $\left(\hat{p}_{\mathrm{opt}}^{*}, \hat{r}_{\mathrm{opt}}^{*}\right)$ are not significant in our consideration of chattering arcs which do not include the singular arc. The condition (43) directly implies upper semicontinuity of the multi-valued right-hand side of the introduced differential inclusion with respect to the vector of the state variables. By virtue of the results from [48, Chapter 2, $\S 8$ ] and [49, Chapter 2, Section 2.2], solutions to this differential inclusion are continuous with respect to initial data. This may serve as a particular theoretical justification for the validity of the proposed method.

\section{Remark 7.2}

One can see that the described method in principle does not depend on a particular finite time horizon $\hat{T}$. 
It is reasonable to validate this numerical approach by testing it on classical Fuller problem $[24,25]$. The corresponding results are presented in Appendix B, and the approximate switching curve fits the theoretical one very well.

\section{NUMERICAL SIMULATIONS}

\subsection{Parameter specification}

Let the dynamic optimization problem (11)-(14) describe resource allocation for a population of Escherichia coli. As in [8], take

$$
\begin{gathered}
\beta=0.003 \mathrm{~L} \cdot \mathrm{g}^{-1}, \quad K_{R}=1 \mathrm{~g} \cdot \mathrm{L}^{-1}, \\
\alpha_{\min }=0, \quad \alpha_{\max }=1 .
\end{gathered}
$$

Set the recycling parameters as

$$
\lambda_{M}=\lambda_{R}=0.8
$$

In compliance with Subsection 2.3, consider the temperature range $\mathcal{T}_{0} \leqslant \mathcal{T} \leqslant \mathcal{T}_{1}$ with the endpoints

$$
\mathcal{T}_{0}=37^{\circ} \mathrm{C}=310.15 \mathrm{~K}, \quad \mathcal{T}_{1}=45^{\circ} \mathrm{C}=318.15 \mathrm{~K} .
$$

The parameters (44),(45) are assumed to be constant within this range. Appendix A explains a way to modify our model in case of a temperature change. According to [19], we choose the following activation energies:

$$
\begin{gathered}
\mathcal{E}_{1}=15 \mathrm{kcal} \cdot \mathrm{mol}^{-1}=62760 \mathrm{~J} \cdot \mathrm{mol}^{-1} \text { for metabolism and gene expression, } \\
\mathcal{E}_{2}=150 \mathrm{kcal} \cdot \mathrm{mol}^{-1}=627600 \mathrm{~J} \cdot \mathrm{mol}^{-1} \text { for degradation. }
\end{gathered}
$$

As introduced in Appendix A, the function $\varphi_{1}$ describes how temperature affects the parameters $e_{M}$ and $k_{R}$ for metabolism and gene expression, the function $\varphi_{2}$ specifies the effect on the degradation rates $\gamma_{M}, \gamma_{R}$, and $\varphi \stackrel{\text { def }}{=} \varphi_{2} / \varphi_{1}$ is the corresponding ratio (see the formulas (53),(54)). Fig. 3 illustrates the graphs of the sections $\varphi_{1}\left(\cdot, \mathcal{T}_{0}\right)$ and $\varphi\left(\cdot, \mathcal{T}_{0}\right)$ on the observed temperature interval. By virtue of the resulting transformation rule (55) and dramatic increase of $\varphi\left(\cdot, \mathcal{T}_{0}\right)$ as compared to $\varphi_{1}\left(\cdot, \mathcal{T}_{0}\right)$, the influence of the degradation rates should become stronger for higher temperatures.

Let the values of the parameters $e_{M}, k_{R}$ from [8] correspond to the temperature $37^{\circ} \mathrm{C}$ :

$$
\left.e_{M}\right|_{\mathcal{T}=\mathcal{T}_{0}}=3.6 \mathrm{~h}^{-1},\left.\quad k_{R}\right|_{\mathcal{T}=\mathcal{T}_{0}}=3.6 \mathrm{~h}^{-1} .
$$



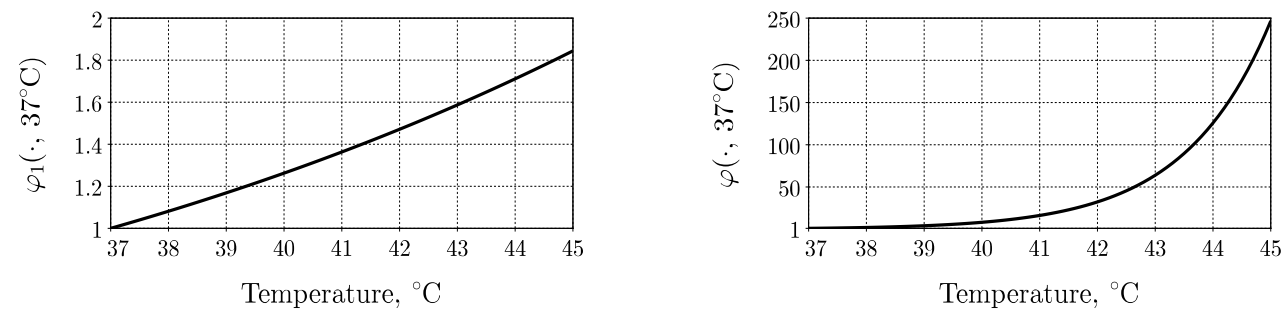

Figure 3. The functions $\varphi_{1}\left(\cdot, \mathcal{T}_{0}\right)$ and $\varphi\left(\cdot, \mathcal{T}_{0}\right)$ for the parameter values (46),(47). Here the Celsius temperature scale is used for convenience.

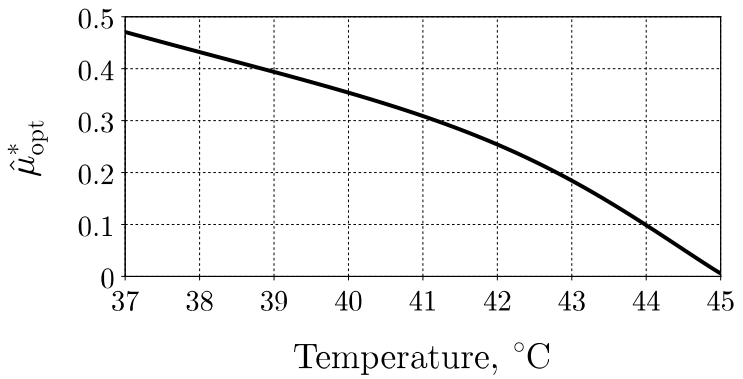

Figure 4. The optimal dimensionless steady-state growth rate $\hat{\mu}_{\mathrm{opt}}^{*}$ depending on temperature for the parameter values (44)-(49). Here the Celsius temperature scale is used for convenience.

In case of this temperature, let us also set the average half-life time of a macromolecule as $20 \mathrm{~h}$ for metabolic machinery and $100 \mathrm{~h}$ for gene expression machinery, i.e.,

$$
\left.\gamma_{M}\right|_{\mathcal{T}=\mathcal{T}_{0}}=\frac{\ln 2}{20} \mathrm{~h}^{-1},\left.\quad \gamma_{R}\right|_{\mathcal{T}=\mathcal{T}_{0}}=\frac{\ln 2}{100} \mathrm{~h}^{-1} .
$$

Such a value of $\gamma_{M}$ conforms with the relevant ranges specified in $[18,36]$. Due to a significantly higher stability of proteins for gene expression in comparison with metabolic ones [17, 18,36-38], here it is reasonable to take $\gamma_{R}$ much smaller than $\gamma_{M}$.

\subsection{Effect of temperature}

Fig. 4 depicts the dependence of the optimal dimensionless steady-state growth rate $\hat{\mu}_{\mathrm{opt}}^{*}$ on temperature. Since the transformation rule (55) contains a time variable change, the growth rates at different temperatures are correctly compared only if they are evaluated with respect to the same time variable $\hat{t}$. We suppose that $\hat{t}$ is related to the system for the temperature $37^{\circ} \mathrm{C}$. For $45^{\circ} \mathrm{C}$, the growth rate appears to be about 100 times lower than for $37^{\circ} \mathrm{C}$. In fact, the particular value of $\left.\gamma_{R}\right|_{\mathcal{T}=\mathcal{T}_{0}}$ in (49) is chosen so as to achieve this good agreement with the experimental results of [19]. It is also important to emphasize that, in the whole considered temperature range, our parameter values satisfy Assumptions 3.1-3.3, 4.1-4.4 and the second inequality of Assumption 5.1. However, the first inequality of Assumption 5.1 holds on the temperature interval from $37^{\circ} \mathrm{C}$ till approximately $44.2^{\circ} \mathrm{C}$, and it is violated for higher temperatures. 


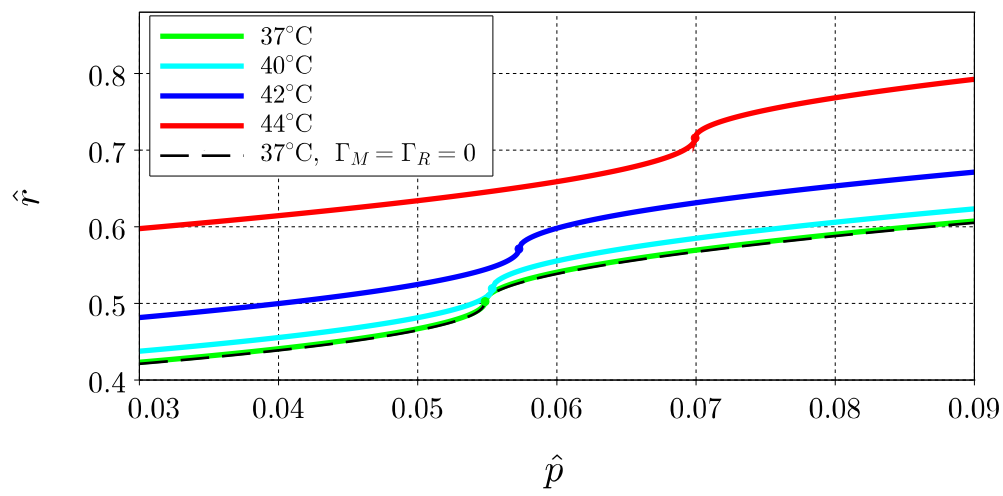

Figure 5. The chattering switching curves for different temperatures in case of the parameter values (44)(49). The curve in case of the temperature $37^{\circ} \mathrm{C}$ and zero degradation rates $\Gamma_{M}=\Gamma_{R}=0$ is also shown for comparison. Here the Celsius temperature scale is used for convenience.

The numerical method of Section 7 is used to approximate the chattering switching curve $\Sigma$ of the optimal feedback control law in the problem (11)-(14). Fig. 5 indicates the dependence of this curve on temperature. It is evident that, as opposed to the degradation rates, the optimal feedback strategy of bacterial resource allocation is low sensitive to temperature changes within the subrange $\left[37^{\circ} \mathrm{C}, 42^{\circ} \mathrm{C}\right]$.

\subsection{Dynamics of optimal processes}

For illustrating the dynamics of the optimal processes, let us fix the temperature

$$
\mathcal{T}=42^{\circ} \mathrm{C}=315.15 \mathrm{~K},
$$

time horizon

$$
\hat{T}=k_{R} \cdot T=10,
$$

and initial position as the optimal steady state for a lower environmental input:

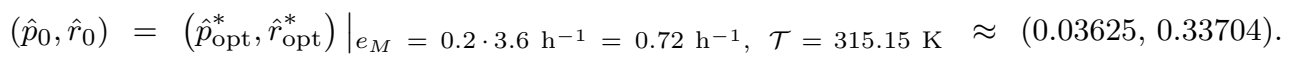

The latter condition can be reasonable, for example, if the bacterial population is considered directly after a nutrient upshift, such as transferring from a poor environment to a richer one with the same temperature. If the time variable $\hat{t}$ is related to the system for the temperature $\mathcal{T}_{0}$, then, according to (55), the new time variable $\hat{\xi}$ is represented as

$$
\hat{\xi}=\varphi_{1}\left(\mathcal{T}, \mathcal{T}_{0}\right) \cdot \hat{t} \approx 1.4713 \cdot \hat{t},
$$

and, due to (51), the new time horizon equals

$$
\hat{\Xi}=\varphi_{1}\left(\mathcal{T}, \mathcal{T}_{0}\right) \cdot \hat{T} \approx 14.713
$$



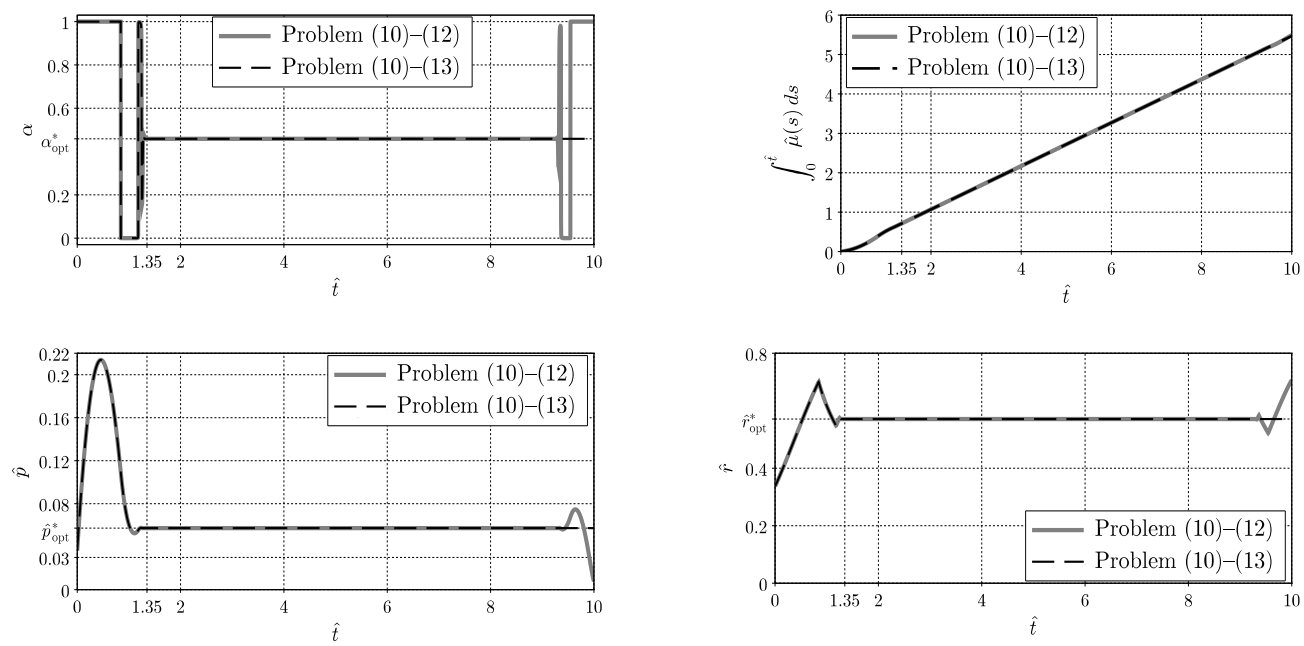

Figure 6. The approximate optimal process computed via Bocop for the problem (11)-(14) with the parameter values (44)-(51) and initial condition (52). For comparison, we also show the approximate optimal process in the corresponding problem (11)-(13) without the terminal condition (14).

Fig. 6 indicates the results of numerical computation of the optimal open-loop control function via the software package Bocop [50] (despite the usage of the transformed system in the computation, the illustrations are provided in the original time scale $\hat{t}$ ). For comparison, we also show the optimal process in the corresponding problem (11)-(13) without the terminal condition (14). As one can see, imposing this condition excludes the final chattering regime (by which the optimal steady state is left) and gives only a slightly lower quality of the optimal control strategy. Fig. 7 depicts the approximate optimal process for the greater time horizon $\hat{T}=20$. By comparing the results for the problem (11)-(14) in the two cases $\hat{T}=10$ and $\hat{T}=20$, we conclude that, on the time subinterval before $\hat{t}=10$, the processes are almost identical, and then the control law in the second case continues to keep the system in the steady-state singular regime till the end. Numerical experiments with greater time horizons have shown a similar situation. Thus, the mentioned properties are practical justifications for our problem statement with the condition (14).

According to Figure 8, the Bocop simulation results are in good agreement with the optimal control synthesis constructed through the numerical method of Section 7.

Finally, Figure 9 indicates that the chattering switching curve is somewhat deformed and shifted to the left and down when the recycling parameters $\lambda_{M}, \lambda_{R}$ are decreased. In particular, more intensive recycling allows to maintain higher steady-state growth.

\section{CONCLUSION}

In this paper, we have extended the bacterial growth models of $[8,17]$ by including both degradation of proteins into amino acids and recycling of the latter (i. e., using as precursors again). The related dynamic optimization problem with the growth maximization criterion has been investigated. The constructed solution in the form of a feedback control law with a chattering switching curve can 

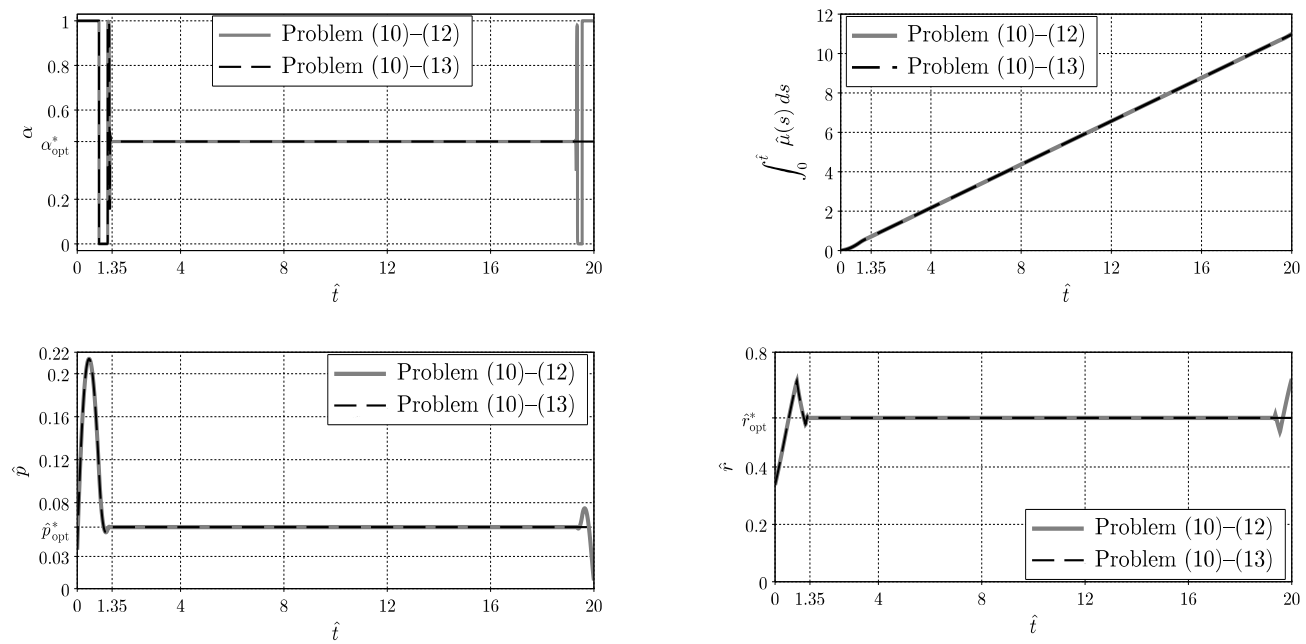

Figure 7. The approximate optimal process computed via Bocop for the problem (11)-(14) with the parameter values (44)-(50), time horizon $\hat{T}=20$ and initial condition (52). For comparison, we also show the approximate optimal process in the corresponding problem (11)-(13) without the terminal condition (14).

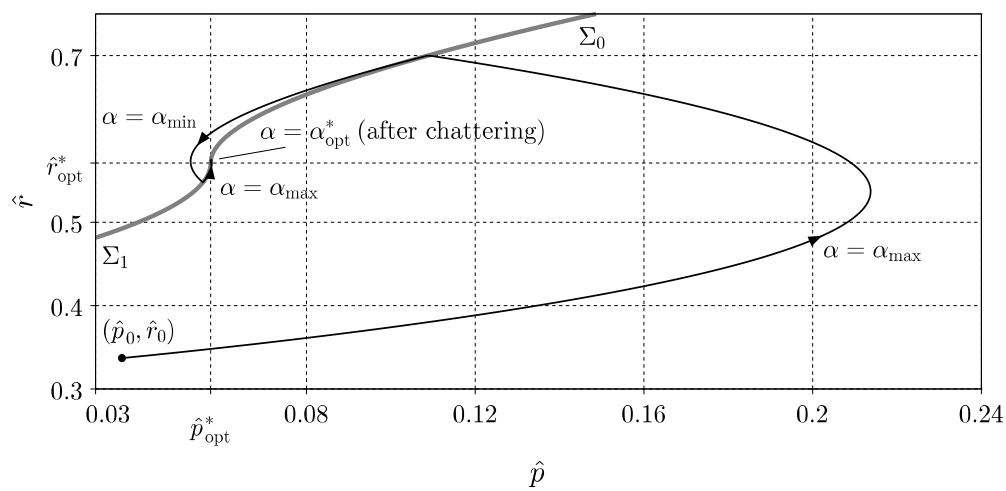

Figure 8. The approximate optimal state trajectory computed via Bocop, and the chattering switching curve constructed through the numerical method of Section 7. The related problem is (11)-(14), the parameter values and initial condition are specified by (44)-(52).

serve as a benchmark for comparing actual bacterial strategies of resource allocation. We have also studied the influence of temperature, whose increase intensifies protein degradation. The optimal feedback control strategy has turned out to be essentially less sensitive to temperature changes, while the growth rate has been deeply affected.

Note that, in comparison with purely numerical techniques, our approach contains a detailed analytical investigation via Pontryagin's maximum principle and thereby allows to obtain important qualitative properties of optimal processes. Moreover, our method to approximate the chattering switching curve relies on these properties. In [51,52], other approaches to compute optimal processes with chattering arcs were developed as applied to minimum time problems for rocket 


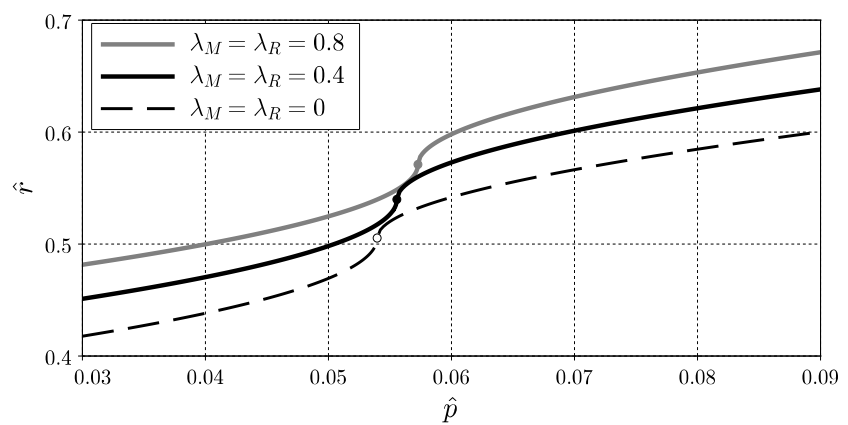

Figure 9. The chattering switching curves for different levels of recycling in case of the parameter values (44), (46)-(50).

and spacecraft dynamic models, and preliminary theoretical analysis by Pontryagin's maximum principle also played a crucial role there.

Besides, it is possible to design suboptimal feedback control policies relying on biological arguments so that the optimal steady state is eventually approached when using "measurements" of either the amounts of nutrients and precursors or the imbalance between the precursors and gene expression machinery [8]. The next step is to evaluate the robustness of these realistic strategies with respect to temperature (the constructed optimal control law can be used as a reference).

One forward-looking extension of our model is to include stochastic uncertainties in the implementation of resource allocation strategies. This will lead to a stochastic optimal control problem with a state-dependent noise and with the criterion of mean growth maximization. Then one might expect that, instead of the switching curve from the deterministic problem, there will appear a transition domain with nonzero Lebesgue measure and intermediate resource allocation. Such a situation was observed, for instance, in the recent studies of [53,54] concerning financial and mechanical stochastic models. Therefore, the stochastic extension of our deterministic problem may lead to some realistic regularization of the chattering control synthesis, while achieving better agreement with experimental approximations of the ppGpp-based strategy in Escherichia coli [7,8]. However, stochastic dynamic optimization problems usually turn out to be substantially more difficult for investigation than deterministic ones [55-57].

Another meaningful further development of our model is to account for the costs of the molecular implementations of bacterial control strategies. Indeed, the latter in general require additional resources to be diverted towards the synthesis of sensory systems and regulatory proteins [26,27].

From the engineering point of view, it is also relevant to extend the model of this paper by introducing the reaction that converts bacterial resources into a particular product of biotechnological interest. Regulations of an external inducer (based on specific chemical or light effects) can modify cellular processes [3]. Then it seems promising to consider the problem of finding an inducing strategy that maximizes the biotechnological production. 


\section{ACKNOWLEDGEMENTS}

This work was supported in part by the project RESET Bioinformatique (ANR-11-BINF-0005) and program LABEX SIGNALIFE (ANR-11-LABX-0028-01). We thank Hidde de Jong, IBIS group at INRIA Grenoble — Rhône-Alpes research center, for enlightening discussions and comments.

\section{REFERENCES}

1. Schaechter, M., Ingraham, J. L., and Neidhardt, F. C. Microbe. ASM Press: Washington, DC, 2006.

2. Venayak, N., Anesiadis, N., Cluett, W. R., and Mahadevan, R. Engineering metabolism through dynamic control. Current Opinion in Biotechnology 2015; 34: 142-152.

3. Izard, J., Gomez Balderas, C. D., Ropers, D., Lacour, S., Song, X., Yang, Y., Lindner, A. B., Geiselmann, J., and de Jong, H. A synthetic growth switch based on controlled expression of RNA polymerase. Molecular Systems Biology 2015; 11(11): 840.

4. Varma, A. and Palsson, B. O. Stoichiometric flux balance models quantitatively predict growth and metabolic byproduct secretion in wild-type Escherichia coli W3110. Applied Environmental Microbiology 1994; 60(10): 3724 3731.

5. Mahadevan, R., Edwards, J. S., and Doyle III, F. J. Dynamic flux balance analysis of diauxic growth in Escherichia coli. Biophysics Journal 2002; 83(3): 1331-1340.

6. Schuetz, R., Zamboni, N., Zampieri, M., Heinemann, M., and Sauer, U. Multidimensional optimality of microbial metabolism. Science 2012; 336(6081): 601-604.

7. Bosdriesz, E., Molenaar, D., Teusink, B., and Bruggeman, F. J. How fast-growing bacteria robustly tune their ribosome concentration to approximate growth-rate maximization. The FEBS Journal 2015; 282: 2029-2044.

8. Giordano, N., Mairet, F., Gouzé, J.-L., Geiselmann, J., and de Jong, H. Dynamical allocation of cellular resources as an optimal control problem: Novel insights into microbial growth strategies. PLoS Computational Biology 2016; 12(3):e1004802. DOI: 10.1371/journal.pcbi.1004802

9. Schuetz, R., Kuepfer, L., and Sauer, U. Systematic evaluation of objective functions for predicting intracellular fluxes in Escherichia coli. Molecular Systems Biology 2007; 3: 119.

10. Goelzer, A., Fromion, V., and Scorletti, G. Cell design in bacteria as a convex optimization problem. Automatica 2011; 47: 1210-1218.

11. O'Brien, E. J., Lerman, J. A., Chang, R. L., Hyduke, D. R., and Palsson, B. O. Genome-scale models of metabolism and gene expression extend and refine growth phenotype prediction. Molecular Systems Biology 2013; 9(1): 693.

12. Waldherr, S., Oyarzún, D. A., and Bockmayr, A. Dynamic optimization of metabolic networks coupled with gene expression. Journal of Theoretical Biology 2015; 365: 469-485.

13. Molenaar, D., van Berlo, R., de Ridder, D., and Teusink, B. Shifts in growth strategies reflect tradeoffs in cellular economics. Molecular Systems Biology 2009; 5: 323.

14. van den Berg, H. A., Kiselev, Yu. N., Kooijman, S. A. L. M., and Orlov, M. V. Optimal allocation between nutrient uptake and growth in a microbial trichome. Journal of Mathematical Biology 1998; 37(1): 28-48.

15. van den Berg, H. A., Kiselev, Yu. N., and Orlov, M. V. Optimal allocation of building blocks between nutrient uptake systems in a microbe. Journal of Mathematical Biology 2002; 44(3): 276-296.

16. Scott, M., Gunderson, C.W., Mateescu, E. M., Zhang, Z., and Hwa, T. Interdependence of cell growth and gene expression: origins and consequences. Science 2010; 330(6007): 1099-1102.

17. Yegorov, I., Mairet, F., and Gouzé, J.-L. Optimal resource allocation for bacterial growth with degradation. IFACPapersOnline 2017; 50(1): 9858-9863.

18. Mandelstam, J. Turnover of protein in growing and non-growing populations of Escherichia coli. Biochemical Journal 1958; 69(1): 110-119.

19. Johnson, F. H. and Lewin, I. The growth rate of E. coli in relation to temperature, quinine and coenzyme. Journal of Cellular Physiology 1946; 28(1): 47-75.

20. Farewell, A. and Neidhardt, F.C. Effect of temperature on in vivo protein synthetic capacity in Escherichia coli. Journal of Bacteriology 1998; 180(17): 4704-4710.

21. Grimaud, G. M., Mairet, F., and Bernard, O. Modelling thermal adaptation in microalgae: an adaptive dynamics point of view. IFAC Proceedings Volumes 2014; 47(3): 4376-4381.

22. Grimaud, G. M., Le Guennec, V., Ayata, S.-D., Mairet, F., Sciandra, A., and Bernard, O. Modelling the effect of temperature on phytoplankton growth across the global ocean. 8th Vienna International Conference on Mathematical 
Modelling - MATHMOD 2015; 48(1): 228-233.

23. Pontryagin, L. S., Boltyansky, V. G., Gamkrelidze, R. V., and Mishchenko, E. F. The Mathematical Theory of Optimal Processes. Macmillan: New York, 1964.

24. Zelikin, M.I. and Borisov, V.F. Theory of Chattering Control with Applications to Astronautics, Robotics, Economics, and Engineering. Birkhauser: Boston, 1994.

25. Schattler, H. and Ledzewicz, U. Geometric Optimal Control: Theory, Methods and Examples. Springer: New York, 2012.

26. Kalisky, T., Dekel, E., and Alon, U. Cost-benefit theory and optimal design of gene regulation functions. Physical Biology 2007; 4(4): 229-245.

27. Poelwijk, F. J., de Vos, M. G., and Tans, S. J. Tradeoffs and optimality in the evolution of gene regulation. Cell 2011; 146(3): 462-470.

28. Makrides, S. C. Strategies for achieving high-level expression of genes in Escherichia coli. Microbiological Reviews 1996; 60(3): 512-538.

29. Ehrenberg, M., Bremer, H., and Dennis, P. P. Medium-dependent control of the bacterial growth rate. Biochimie 2013; 95(4): 643-658.

30. Carlson, D. A., Haurie, A. B., and Leizarowitz, A. Infinite Horizon Optimal Control: Deterministic and Stochastic Systems. Springer-Verlag: Berlin, Heidelberg, 1991.

31. Trélat, E. and Zuazua, E. The turnpike property in finite-dimensional nonlinear optimal control. Journal of Differential Equations 2015; 258(1):81-114.

32. Clark, C. W. Mathematical Bioeconomics: The Optimal Management of Renewable Resources. John Wiley \& Sons: New York, 1990.

33. Laidler, K. J. Chemical Kinetics. Third edition. Harper and Row: New York, 1987.

34. Clarke, F. H., Ledyaev, Yu. S., Stern, R. J., and Wolenski, P. R. Nonsmooth Analysis and Control Theory. SpringerVerlag: New York, 1998.

35. Lakshmikantham, V. and Leela, S. Differential and Integral Inequalities: Theory and Applications. Volume I: Ordinary Differential Equations. Academic Press: New York, London, 1969.

36. Borek, E., Ponticorvo, L., and Rittenberg, D. Protein turnover in micro-organisms. Proceedings of the National Academy of Sciences of the United States of America 1958; 44(5): 369-374.

37. Neidhardt, F. C. The regulation RNA synthesis in bacteria. Progress in Nucleic Acid Research and Molecular Biology 1964; 3: 145-181.

38. Deutscher, M. P. Degradation of stable RNA in bacteria. Journal of Biological Chemistry 2003; 278: 45041-45044.

39. Afanas'ev, V. N., Kolmanovskii, V. B., and Nosov, V. R. Mathematical Theory of Control Systems Design. Kluwer Academic Publishers: Dordrecht, 1996.

40. Lee, E. B. and Markus L. Foundations of Optimal Control Theory. Krieger Publishing: Malabar, 1986.

41. Perko, L. Differential Equations and Dynamical Systems. Springer-Verlag: New York, 2001.

42. Gabasov, R. and Kirillova, F. M. Singular Optimal Control. Plenum Press: New York, 1982.

43. Melikyan, A. A. Generalized Characteristics of First Order PDEs: Application in Optimal Control and Differential Games. Birkhauser: Boston, 1998.

44. Bonnard, B. and Chyba, M. Singular Trajectories and their Role in Control Theory. Mathématiques \& Applications, 40. Springer-Verlag: Paris, 2003.

45. Zelikin, M.I. and Borisov, V.F. Singular optimal regimes in problems of mathematical economics. Journal of Mathematical Sciences 2005; 130(1): 4409-4570.

46. Schattler, H. and Ledzewicz, U. Optimal Control for Mathematical Models of Cancer Therapies: An Application of Geometric Methods. Interdisciplinary Applied Mathematics, 42. Springer-Verlag: New York, 2015.

47. Naumov, G. V. Construction of the switching curve for optimal control problems with chattering control. Izvestiya RAN: Teoriya i Sistemy Upravleniya 2003; 3: 46-51 (in Russian).

48. Filippov, A. F. Differential Equations with Discontinuous Right-hand Sides. Kluwer Academic: Dordrecht, 1988.

49. Blagodatskikh, V.I. and Filippov, A.F. Differential inclusions and optimal control. Proceedings of the Steklov Institute of Mathematics 1986; 169: 199-259.

50. Bonnans, F., Martinon, P., Giorgi, D., Grélard, V., Maindrault, S., Tissot, O., and Liu, J. Bocop 2.0.5 — User Guide. February 8, 2017. URL: http://bocop.org

51. Zhu, J., Trélat, E., and Cerf, M. Minimum time control of the rocket attitude reorientation associated with orbit dynamics. SIAM Journal on Control and Optimization 2016; 54(1): 391-422.

52. Zhu, J., Trélat, E., and Cerf, M. Planar tilting maneuver of a spacecraft: singular arcs in the minimum time problem and chattering. Discrete and Continuous Dynamical Systems - Series B 2016; 21(4): 1347-1388.

53. Bratus, A., Yegorov, I., and Yurchenko, D. Optimal investment strategies in a certain class of stochastic Merton's terminal wealth problems. International Journal of Dynamics and Control 2016; 5(3): 771-782. 
54. Bratus, A., Yegorov, I., and Yurchenko, D. Optimal bounded noisy feedback control for damping random vibrations. Journal of Vibration and Control 2016. DOI: 10.1177/1077546316670072

55. Kolosov, G. E. Optimal Design of Control Systems: Stochastic and Deterministic Problems. Marcel Dekker: New York, Basel, 1999.

56. Yong, J. and Jou, X. Yu. Stochastic Controls: Hamiltonian Systems and HJB Equations. Springer-Verlag: New York, 1999.

57. Fleming, W. H. and Soner, H. M. Controlled Markov Processes and Viscosity Solutions. Springer-Verlag: New York, 2006.

\section{A. EFFECT OF TEMPERATURE}

A classical way to describe the dependence of the rate of a chemical reaction on temperature $\mathcal{T}[\mathrm{K} \equiv$ Kelvin] is to use Arrhenius law [33]

$$
\text { Reaction rate parameter }=\mathcal{A} \cdot \exp \left(-\frac{\mathcal{E}}{\mathcal{R} \mathcal{T}}\right)
$$

with suitable pre-exponential factor $\mathcal{A}$ (the related unit of measurement may vary) and activation energy $\mathcal{E}\left[\mathrm{J} \cdot \mathrm{mol}^{-1}\right]$. Here $\mathcal{R} \approx 8.314 \mathrm{~J} \cdot \mathrm{mol}^{-1} \cdot \mathrm{K}^{-1}$ is the ideal gas constant, and calligraphy letters for the variables $\mathcal{A}, \mathcal{E}, \mathcal{R}, \mathcal{T}$ are used so as to avoid confusion with earlier notations.

Consider growth and degradation processes for Escherichia coli in the temperature range $\left[\mathcal{T}_{0}, \mathcal{T}_{1}\right]=\left[37^{\circ} \mathrm{C}, 45^{\circ} \mathrm{C}\right]=[310.15 \mathrm{~K}, 318.15 \mathrm{~K}]$. In conformity with $[19,20]$, here it is reasonable to employ Arrhenius law and the fact that protein degradation appears to be more sensitive to temperature changes as compared to metabolism and gene expression. Let $\mathcal{E}_{1}$ be the activation energy for both metabolism and gene expression. Then the activation energy for degradation is $\mathcal{E}_{2}>\mathcal{E}_{1}$.

Assume that the parameters $\beta, K_{R}, \alpha_{\min }, \alpha_{\max }, \lambda_{M}, \lambda_{R}$ are constant in the whole considered temperature range. Let the rates $e_{M}, k_{R}, \gamma_{M}, \gamma_{R}$ correspond to some temperature $\mathcal{T} \in\left[\mathcal{T}_{0}, \mathcal{T}_{1}\right]$. For another temperature $\tilde{\mathcal{T}} \in\left[\mathcal{T}_{0}, \mathcal{T}_{1}\right]$, Arrhenius law leads to the modified parameters

$$
\begin{aligned}
& \tilde{e}_{M}=\varphi_{1}(\tilde{\mathcal{T}}, \mathcal{T}) \cdot e_{M}, \quad \tilde{k}_{R}=\varphi_{1}(\tilde{\mathcal{T}}, \mathcal{T}) \cdot k_{R}, \\
& \tilde{\gamma}_{M}=\varphi_{2}(\tilde{\mathcal{T}}, \mathcal{T}) \cdot \gamma_{M}, \quad \tilde{\gamma}_{R}=\varphi_{2}(\tilde{\mathcal{T}}, \mathcal{T}) \cdot \gamma_{R},
\end{aligned}
$$

where

$$
\varphi_{i}(\tilde{\mathcal{T}}, \mathcal{T})=\exp \left(\frac{\mathcal{E}_{i}}{\mathcal{R}}\left(\frac{1}{\mathcal{T}}-\frac{1}{\tilde{\mathcal{T}}}\right)\right), \quad i=1,2
$$

and, moreover,

$$
\tilde{v}_{M}(r(t))=\varphi_{1}(\tilde{\mathcal{T}}, \mathcal{T}) \cdot v_{M}(r(t)), \quad \tilde{v}_{R}(p(t), r(t))=\varphi_{1}(\tilde{\mathcal{T}}, \mathcal{T}) \cdot v_{R}(p(t), r(t))
$$

(recall the representations (6) and (7)). Also denote

$$
\varphi(\tilde{\mathcal{T}}, \mathcal{T}) \stackrel{\text { def }}{=} \frac{\varphi_{2}(\tilde{\mathcal{T}}, \mathcal{T})}{\varphi_{1}(\tilde{\mathcal{T}}, \mathcal{T})}=\exp \left(\frac{\mathcal{E}_{2}-\mathcal{E}_{1}}{\mathcal{R}}\left(\frac{1}{\mathcal{T}}-\frac{1}{\tilde{\mathcal{T}}}\right)\right) .
$$


Since $\mathcal{E}_{2}>\mathcal{E}_{1}$, this expression strictly increases with respect to $\tilde{\mathcal{T}}$ for a fixed $\mathcal{T}$.

One can easily verify that, for the temperature $\tilde{\mathcal{T}}$, it suffices to modify the system (5) by the direct replacements

$$
\begin{gathered}
t \longrightarrow \xi \stackrel{\text { def }}{=} \varphi_{1}(\tilde{\mathcal{T}}, \mathcal{T}) \cdot t, \\
T \longrightarrow \Xi \stackrel{\text { def }}{=} \varphi_{1}(\tilde{\mathcal{T}}, \mathcal{T}) \cdot T, \\
\gamma_{M} \longrightarrow \tilde{\tilde{\gamma}}_{M} \stackrel{\text { def }}{=} \varphi(\tilde{\mathcal{T}}, \mathcal{T}) \cdot \gamma_{M}, \\
\gamma_{R} \longrightarrow \tilde{\tilde{\gamma}}_{R} \stackrel{\text { def }}{=} \varphi(\tilde{\mathcal{T}}, \mathcal{T}) \cdot \gamma_{R} .
\end{gathered}
$$

Therefore, the problem (11)-(14) in dimensionless variables should be modified by the replacements

$$
\begin{gathered}
\hat{t} \longrightarrow \hat{\xi} \stackrel{\text { def }}{=} \varphi_{1}(\tilde{\mathcal{T}}, \mathcal{T}) \cdot \hat{t}, \\
\hat{T} \longrightarrow \hat{\Xi} \stackrel{\text { def }}{=} \varphi_{1}(\tilde{\mathcal{T}}, \mathcal{T}) \cdot \hat{T}, \\
\Gamma_{M} \longrightarrow \tilde{\tilde{\Gamma}}_{M} \stackrel{\text { def }}{=} \varphi(\tilde{\mathcal{T}}, \mathcal{T}) \cdot \Gamma_{M}, \\
\Gamma_{R} \longrightarrow \tilde{\tilde{\Gamma}}_{R} \stackrel{\text { def }}{=} \varphi(\tilde{\mathcal{T}}, \mathcal{T}) \cdot \Gamma_{R} .
\end{gathered}
$$

Thus, for higher temperatures, the form of our dynamical system remains similar, but the influence of the degradation rates increases.

\section{B. FULLER PROBLEM}

Fuller problem is stated as follows [24,25]:

$$
\left\{\begin{array}{l}
\frac{d x_{1}(t)}{d t}=x_{2}(t), \\
\frac{d x_{2}(t)}{d t}=u(t), \\
-1 \leqslant u(t) \leqslant 1, \quad t \in[0, T], \quad T \in[0,+\infty) \text { is free, } \\
\left(x_{1}(0), x_{2}(0)\right)=\left(x_{1}^{0}, x_{2}^{0}\right) \in \mathbb{R}^{2} \text { is fixed, } \\
\left(x_{1}(T), x_{2}(T)\right)=(0,0), \\
\frac{1}{2} \int_{0}^{T} x_{1}^{2}(t) d t \longrightarrow \inf .
\end{array}\right.
$$

The next result gives its analytical solution in the feedback form (the proof can be found in $[24,25]$ ).

Theorem B.1

Let $\zeta \stackrel{\text { def }}{=} \sqrt{(\sqrt{33}-1) / 24}=0.4446236 \ldots$ be the unique positive root of the equation $z^{4}+$ 


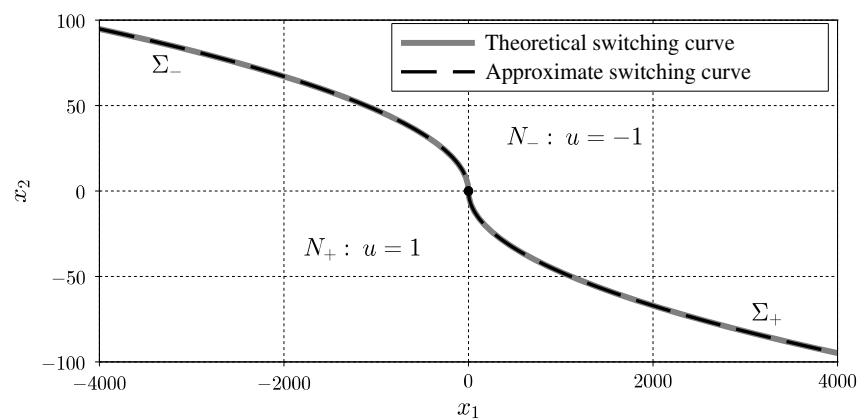

Figure 10. Comparison between the theoretical switching curve $\Sigma$ in Fuller problem (56) and its approximation obtained by using the numerical approach of Section 7.

$z^{2} / 12-1 / 18=0$, and introduce the sets

$$
\begin{gathered}
\Sigma_{-} \stackrel{\text { def }}{=}\left\{\left(x_{1}, x_{2}\right) \in \mathbb{R}^{2}: x_{1}=-\zeta x_{2}^{2}, x_{2}>0\right\}, \\
\Sigma_{+} \stackrel{\text { def }}{=}\left\{\left(x_{1}, x_{2}\right) \in \mathbb{R}^{2}: x_{1}=\zeta x_{2}^{2}, x_{2}<0\right\}, \\
N_{-} \stackrel{\text { def }}{=}\left\{\left(x_{1}, x_{2}\right) \in \mathbb{R}^{2}: x_{1}>-\operatorname{sign}\left(x_{2}\right) \zeta x_{2}^{2}\right\}, \\
N_{+} \stackrel{\text { def }}{=}\left\{\left(x_{1}, x_{2}\right) \in \mathbb{R}^{2}: x_{1}<-\operatorname{sign}\left(x_{2}\right) \zeta x_{2}^{2}\right\} .
\end{gathered}
$$

Then the optimal feedback control law in Fuller problem (56) can be represented as

$$
u_{\text {opt }}\left(x_{1}, x_{2}\right)= \begin{cases}-1, & \left(x_{1}, x_{2}\right) \in N_{-} \cup \Sigma_{-}, \\ 1, & \left(x_{1}, x_{2}\right) \in N_{+} \cup \Sigma_{+} .\end{cases}
$$

The related forward-time state trajectories are chattering arcs. They switch in the direction from $u=-1$ to $u=1$ on $\Sigma_{+}$and in the opposite direction on $\Sigma_{-}$. The curves $\Sigma_{+}$and $\Sigma_{-}$are transversally crossed by the chattering arcs.

Fig. 10 compares the theoretical switching curve $\Sigma \stackrel{\text { def }}{=} \Sigma_{-} \cup\{0,0\} \cup \Sigma_{+}$with its approximation obtained by using the numerical approach of Section 7. One can indeed see a very good agreement there. 